\title{
Hirschsprung-like disease is exacerbated by reduced de novo GMP synthesis
}

\author{
Jonathan I. Lake, ${ }^{1}$ Olga A. Tusheva, ${ }^{1}$ Brittany L. Graham, ${ }^{1}$ and Robert O. Heuckeroth ${ }^{1,2}$
}

${ }^{1}$ Department of Pediatrics and ${ }^{2}$ Department of Developmental, Regenerative and Stem Cell Biology, Washington University School of Medicine, St. Louis, Missouri, USA.

\begin{abstract}
Hirschsprung disease (HSCR) is a partially penetrant oligogenic birth defect that occurs when enteric nervous system (ENS) precursors fail to colonize the distal bowel during early pregnancy. Genetic defects underlie HSCR, but much of the variability in the occurrence and severity of the birth defect remain unexplained. We hypothesized that nongenetic factors might contribute to disease development. Here we found that mycophenolate, an inhibitor of de novo guanine nucleotide biosynthesis, and 8 other drugs identified in a zebrafish screen impaired ENS development. In mice, mycophenolate treatment selectively impaired ENS precursor proliferation, delayed precursor migration, and induced bowel aganglionosis. In 2 different mouse models of HSCR, addition of mycophenolate increased the penetrance and severity of Hirschsprung-like pathology. Mycophenolate treatment also reduced ENS precursor migration as well as lamellipodia formation, proliferation, and survival in cultured enteric neural crest-derived cells. Using $\mathrm{X}$-inactivation mosaicism for the purine salvage gene $\mathrm{Hprt}$, we found that reduced ENS precursor proliferation most likely causes mycophenolate-induced migration defects and aganglionosis. To the best of our knowledge, mycophenolate is the first medicine identified that causes major ENS malformations and Hirschsprung-like pathology in a mammalian model. These studies demonstrate a critical role for de novo guanine nucleotide biosynthesis in ENS development and suggest that some cases of HSCR may be preventable.
\end{abstract}

\section{Introduction}

Hirschsprung disease (HSCR) is a common birth defect ( 1 in 5,000 incidence) in which the enteric nervous system (ENS) is missing from distal bowel (aganglionosis). Because the ENS controls intestinal motility, HSCR causes severe constipation, abdominal distension, bilious vomiting, growth failure, and life-threatening infection (1). Survival requires surgical excision of aganglionic bowel. After surgery, however, enterocolitis (i.e., bowel inflammation) occurs commonly (35\% incidence), and approximately $5 \%$ of affected children still die from HSCR. Infrequently, long-segment HSCR necessitates intravenous nutrition, with accompanying life-threatening infections. Most children with HSCR (80\%) have only a short segment of aganglionosis, which suggests that slightly enhanced bowel colonization by ENS precursors could prevent disease. New strategies are needed to enhance bowel colonization by ENS precursors and to reduce HSCR occurrence.

While HSCR undoubtedly requires genetic defects (2), almost all predisposing mutations have partial penetrance and variable expressivity. For example, inactivating RET mutations occur in $15 \%-20 \%$ of sporadic and $50 \%$ of familial HSCR, but only about half of children with inactivating RET mutations have HSCR (2). Genetic interactions also influence HSCR risk (3-6); however, much variability in occurrence and severity of HSCR and other human birth defects remains unexplained. We hypothesized that nongenetic factors might affect HSCR occurrence. If so, then some cases of HSCR might be preventable by changes in prenatal care.

HSCR is caused by failure of distal bowel colonization by enteric neural crest-derived cells (ENCDCs) during weeks 4-7 of human gestation (7-9). Normally, ENCDCs proliferate vigorously and migrate rostrocaudally to colonize the entire intestine. A similar

Conflict of interest: The authors have declared that no conflict of interest exists. Citation for this article: J Clin Invest. 2013;123(11):4875-4887. doi:10.1172/JCI69781. process occurs in mice from E9.5 to E13.5 and in zebrafish from 36 to 96 hours post fertilization (hpf). Many genes $(7,10)$ in addition to RET are needed for ENS development, including SOX10, $P H O X 2 B, E D N R B, E D N 3, G D N F$, intracellular signaling molecules $(11,12)$, cytoskeletal components $(13,14)$, and adhesion proteins (15-17). $30 \%$ of children with HSCR have additional birth defects, and at least 30 genetic syndromes are HSCR associated (2). The diverse molecular mechanisms supporting ENS development suggest that many nongenetic factors could influence ENCDC bowel colonization and HSCR occurrence by modifying the activity or abundance of needed molecules. Furthermore, chemical perturbation of the developing ENS may identify new pathways involved in ENCDC migration, self renewal, proliferation, or survival.

In order to identify medicines that might increase HSCR risk, gain new insight into HSCR genetics, and identify new modulators of ENS developmental biology, we conducted a zebrafish chemical screen. Here we show that mycophenolic acid (MPA), a commonly used immunosuppressant, caused ENS developmental defects in fish and impaired ENCDC colonization of the bowel in mice via inhibition of inosine monophosphate dehydrogenase (IMPDH), the rate-limiting enzyme in de novo GMP synthesis. Guanine nucleotides are essential for DNA replication and transcription and for >200 GTP-dependent proteins. Our findings suggested that reduced ENCDC proliferation after GTP depletion is the primary cause of MPA-induced bowel aganglionosis (Supplemental Figure 1; supplemental material available online with this article; doi:10.1172/JCI69781DS1) and showed that IMPDH inhibition greatly increased the penetrance and severity of genetic defects affecting the ENS. These studies reinforce the central role of ENCDC proliferation in bowel colonization and raise the intriguing possibility that drugs, nutritional deficiencies, or gene polymorphisms that reduce cell proliferation during early pregnancy may increase HSCR occurrence. 


\section{Table 1}

Compounds that inhibited ENS development in zebrafish

\begin{tabular}{|c|c|c|c|c|c|}
\hline Compound & $\mathrm{TD}_{50}$ in zebrafish ENS & $\mathrm{C}_{\max }$ in mammals & Indication & Primary mechanism & Target molecule \\
\hline Artesunate & $500 \mathrm{nM}$ & $1-3 \mu \mathrm{M}$ & Antimalarial & Unknown & Unknown \\
\hline Benzbromarone & $600 \mathrm{nM}$ & $6-8 \mu \mathrm{M}$ & Uricosuric & Inhibits uric acid reabsorption & URAT1 \\
\hline Cinchophen ${ }^{A}$ & $<100 \mathrm{nM}$ & Unknown & Analgesic/antiinflammatoryB & Possibly cyclooxygenase inhibition & Unknown \\
\hline Closantel & $1.8 \mu \mathrm{M}$ & $82 \mu \mathrm{M}$ & Antihelminthic ${ }^{B}$ & Possibly mitochondrial uncoupling & Unknown \\
\hline Diclazuril & $1 \mu \mathrm{M}$ & $5 \mu \mathrm{M}$ & Coccidiostat ${ }^{B}$ & Unknown & Unknown \\
\hline Flubendazole & $2 \mu \mathrm{M}$ & $20 \mathrm{nM}$ & Antihelminthic ${ }^{B}$ & Inhibits microtubule assembly & $\beta$-tubulinc \\
\hline Lovastatin & $0.4 \mu \mathrm{M}$ & $0.1 \mu \mathrm{M}$ & Antihyperlipidemic & Inhibits cholesterol biosynthesis & HMG-CoA reductase \\
\hline MPA & $1 \mu \mathrm{M}$ & $1-10 \mu \mathrm{M}$ & Immunosuppressant & Inhibits de novo GMP synthesis & IMPDH \\
\hline Oxibendazole & $200 \mathrm{nM}$ & $30 \mathrm{nM}$ & Antihelminthic ${ }^{B}$ & Inhibits microtubule assembly & $\beta$-tubulinc \\
\hline
\end{tabular}

ADose-response studies not performed. ${ }^{\mathrm{B} V}$ eterinary. ${ }^{\mathrm{C}}$ Invertebrate.

\section{Results}

Medications that inhibit ENS development. Zebrafish were treated with 1,508 individual drugs from the Johns Hopkins Clinical Compound Library (Supplemental Table 1 and ref. 18) for the entire duration of ENCDC colonization of the bowel (34-96 hpf). Using $\mathrm{HuC} / \mathrm{HuD}$ (also known as Elavl3/4) immunohistochemistry to visualize neurons (19), we identified 9 compounds that consistently impaired ENS development, were systemically administered, and were not overtly toxic at doses affecting the ENS (Table 1). Dose-response studies permitted determination of median toxic dose $\left(\mathrm{TD}_{50}\right)$ for ENS defects for these drugs. We analyzed MPA in more detail because it dramatically impaired fish ENS development (Figure 1, A-C), is commonly used in humans, has a well-understood molecular mechanism, and has a $\mathrm{TD}_{50}$ within human therapeutic levels (20).

MPA impaired mammalian ENS development. MPA inhibits IMPDH, a protein detected in all E10.5-E12.5 bowel cells (Supplemental Figure 2) that is slightly more abundant in ENCDCs than in neighboring mesenchyme. To determine whether MPA impairs mouse ENS development, we injected pregnant dams with MPA daily from E10.5 through E12.5 and analyzed fetal bowel at E13.5. Since we expected MPA metabolism and effects to vary depending on genetic background, we used both the outbred CF1 strain, due to its high fertility and large litters, and the C57BL/6 standard laboratory strain (referred to herein as B6; see Methods for all complete strain names). CF1 fetuses tolerated $100 \mathrm{mg} / \mathrm{kg} / \mathrm{d}$ (36\% of adult human therapeutic dose, allometrically scaled to mice; ref. 21), but no B6 fetuses survived doses exceeding $25 \mathrm{mg} / \mathrm{kg} / \mathrm{d}$. In both strains, MPA caused dose-dependent reductions in the extent of ENCDC colonization of the bowel (Figure 1, D and E), as indicated by the extent of $\mathrm{TuJ} 1^{+}$neurites in the colon. While we have previously demonstrated that the caudal extent of TuJ1 reactivity indicates the position of the ENCDC wavefront (12), these structures might not be tightly associated after MPA treatment. To directly assess the position of the wavefront, we mated B 6 females to Wnt1-Cre Rosa2 $6^{\text {EYFP/EYFP }}$ males, marking all neural crest-derived cells with enhanced yellow fluorescent protein (EYFP). We examined the position of the most distal staining in the bowel using EYFP, TuJ1, and SOX10 immunoreactivity. Both SOX10 and TuJ1 reliably reflected the extent of bowel colonization seen with EYFP after control or MPA treatment (Supplemental Figure 3), confirming that MPA impaired ENCDC colonization (Figure 1F). While MPA treatment reduced fetal size and colon length (Supplemental Figure 3), it also increased the absolute length of uncolonized colon, suggesting a greater effect of MPA on ENS development than on colon growth.

MPA impaired development of cultured ENCDCs. MPA could reduce migration in vivo by direct effects on ENCDCs or indirect effects on other fetal cells. To study ENCDCs moving on an acellular, migration-permissive surface, we cultured E12.5 midgut slices on fibronectin (12). MPA drastically reduced $\mathrm{RET}^{+}$cell migration out of bowel explants (Figure 2, A, B, and E) and reduced the percentage of migrating ENCDCs with lamellipodia (Figure 2, F-I). MPA also significantly reduced DNA synthesis (Figure 2, A, B, and D) and induced ENCDC apoptosis (Supplemental Figure 4). MPA did not, however, alter neurite growth in postmitotic enteric neurons $\left(\mathrm{BrdU}^{-} \mathrm{TuJ} 1^{+}\right)$grown in low-density dissociated cell culture (Supplemental Figure 5 and ref. 22). To confirm that MPA effects were due to reduced guanine nucleotide levels, we treated cells with guanosine to restore GTP through the purine salvage pathway. Guanosine efficiently rescued MPA's effects on ENCDC migration (Figure 2, C and E), DNA synthesis (Figure 2D), and lamellipodia (Figure 2, F and J), which indicated that the effects of MPA are caused by IMPDH inhibition.

MPA selectively reduced ENCDC DNA synthesis in vivo. To deliver MPA more consistently than drug injections permit, the prodrug mycophenolate mofetil (MMF) was given in drinking water at $1 \mathrm{mg} / \mathrm{ml}$, a dose that improves survival in a mouse lupus model (23). B6 females mated to Wnt1-Cre Rosa26 EYFP/EYFP males were treated with MMF from E10.5 to E13.5 and injected with BrdU 1 hour prior to analysis. MMF decreased distal colon colonization by ENCDCs (Figure 3, A-D), recapitulating the effect of injected MPA. SOX10 and RET immunohistochemistry showed that neuronal differentiation in littermate fetuses was unaffected by MMF (Supplemental Figure 6, A-C). Since MPA might affect transcription of mesenchyme-derived signals required for colonization of the bowel, we measured Gdnf, Edn3, and Ece1 mRNA levels in E13.5 bowel using quantitative RT-PCR (qRT-PCR), but found no differences (Supplemental Figure 6D). In contrast to our in vitro data, cleaved caspase- $3^{+}$cells were rare in both ENCDCs and surrounding mesenchyme after MMF treatment (Supplemental Figure 7), but were readily detected in limb bud interdigital web. These results agree with recent studies that showed low but detectable rates of nuclear fragmentation (24) and cleaved caspase-3 (25) reactivity in WT ENCDCs. While ENCDCs also can die through unconventional, caspase-independent processes in circumstances such as partial loss of RET expression (26), ENCDCs in culture readily undergo canonical apoptosis in response to 

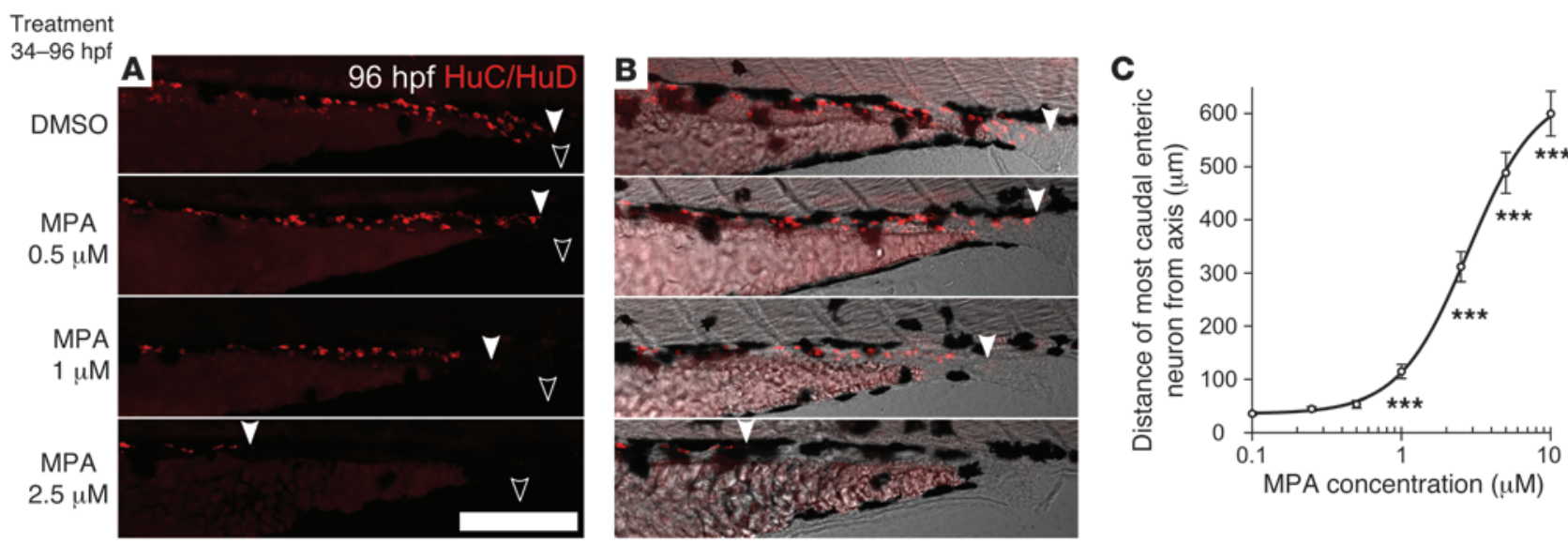

Treatment

E10.5-12.5
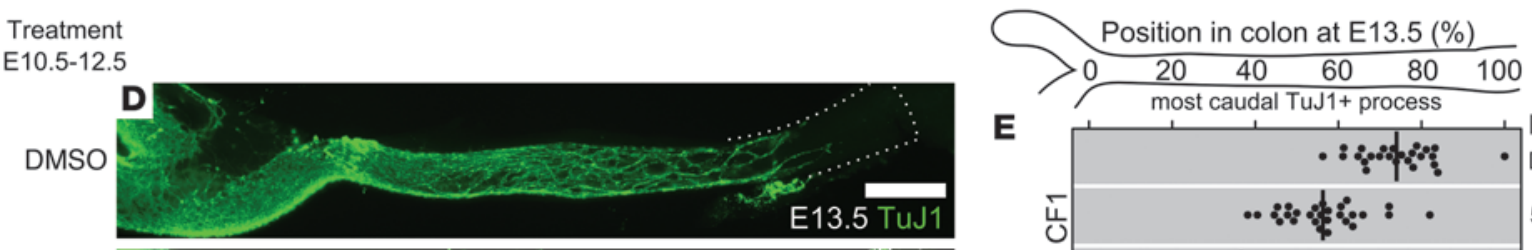

$\mathbf{E}$
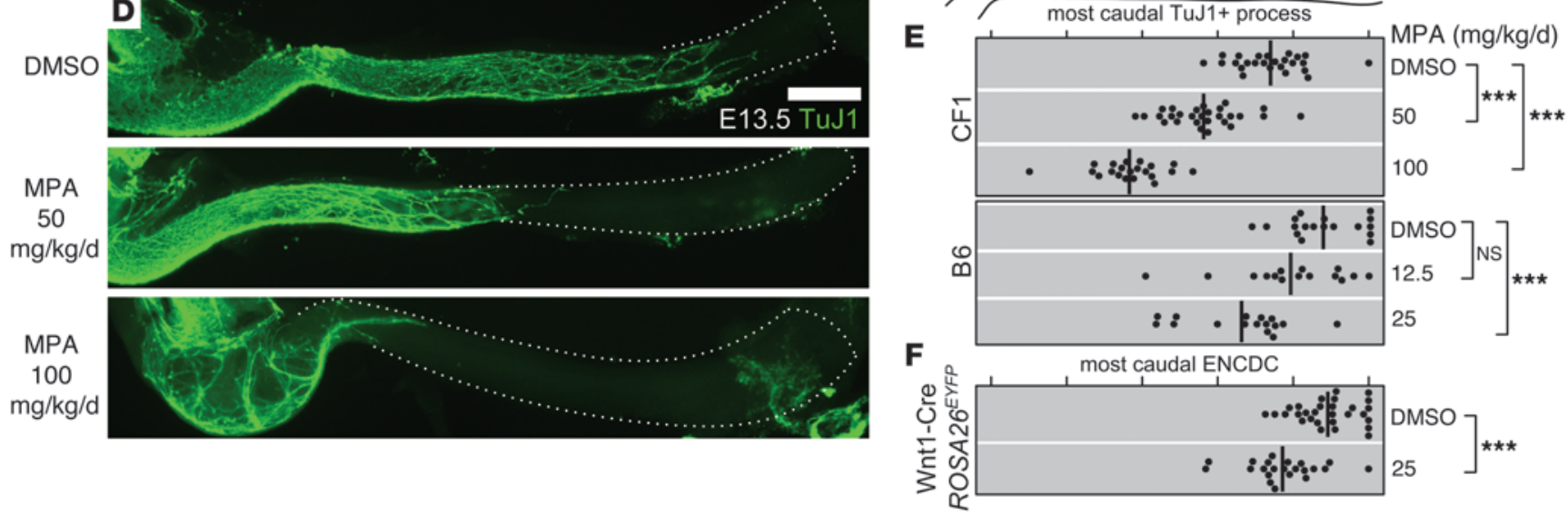

\section{Figure 1}

MPA inhibited ENS development in developing zebrafish and mouse. (A-C) Developing WT zebrafish were exposed to DMSO or MPA from 34 to 96 hpf. (A) Larvae ( $>200)$ were immunostained for neuronal marker HuC/HuD. (B) Images in A merged with transmitted light. Filled arrowheads denote most caudal enteric neuron; open arrowheads denote vents. (C) Average uncolonized distal intestine, plotted vs. MPA dose and compared with control. (D-F) MPA exposure by maternal intraperitoneal injection from E10.5 to E12.5 impaired enteric neuron colonization of the mouse hindgut at E13.5 (D), as visualized by the neuronal marker TuJ1 (left side, ileocecal junction; dotted line, colon outline). The position within each E13.5 colon of the most caudal (E) neuronal process (marked by TuJ1) or (F) ENCDC cell body (ascertained by the lineage marker EYFP or by SOX10 staining in EYFP- littermates) in each E13.5 fetus is plotted for each MPA dose and mouse strain (thick lines denote mean). Scale bars: $250 \mu \mathrm{m}(\mathbf{A}$ and $\mathbf{B}) ; 1 \mathrm{~mm}(\mathbf{D}) .{ }^{* *} P<0.001$, Kolmogorov-Smirnov test $(\mathbf{C})$; ANOVA and $t$ test $(\mathbf{E}$ and $\mathbf{F})$.

MPA, so we limited our examination of cell death to cleaved caspase-3. Consistent with the effects of MPA on ENCDCs in culture, MMF reduced proliferation of colon ENCDCs. In contrast to its effects on ENCDCs, MMF actually increased the fraction of surrounding mesenchymal cells incorporating BrdU (Figure 3, $\mathrm{E}-\mathrm{G})$. Since this was unexpected, given the reduction in bowel size resulting from either MPA or MMF treatment (Supplemental Figure 3 ), we counted mitotic figures within these populations. The mitotic index was reduced within ENCDCs (Figure $3 \mathrm{H}$ ), in concordance with the reduced BrdU incorporation. In contrast to the BrdU results, however, the mitotic index in the mesenchyme was not increased, which indicates that MMF-treated mesenchymal cells entered S-phase, but did not divide at elevated rates. MMF therefore selectively reduced ENCDC proliferation and distal bowel colonization in vivo without increasing caspase-mediated apoptosis or altering neuronal differentiation.
$M P A$ reduced ENCDC migration by reducing proliferation. Guanine nucleotide depletion must underlie MPA's effects, because guanosine rescues these effects in vitro. GTP is essential for DNA, RNA, and protein synthesis as well as for GTPases and many other proteins. To distinguish between primary effects of guanine nucleotide depletion on cell motility versus effects on ENCDC proliferation that secondarily reduce distal bowel colonization, we determined which effects of GTP depletion are cell autonomous. This is important because either reduced ENCDC proliferation or reduced cell intrinsic motility could prevent ENCDC migration into distal bowel (27). We used mice with a null mutation in the purine salvage gene Hprt (28), which is required for guanosine to rescue GTP depletion (29). Since Hprt is X-linked, 1 copy of the Hprt locus is randomly inactivated in each cell of a female. Thus, in all Hprt male cells and in half of $H p r t^{+/-}$female cells, HPRT protein is absent, and guanosine supplementation will not rescue GTP 

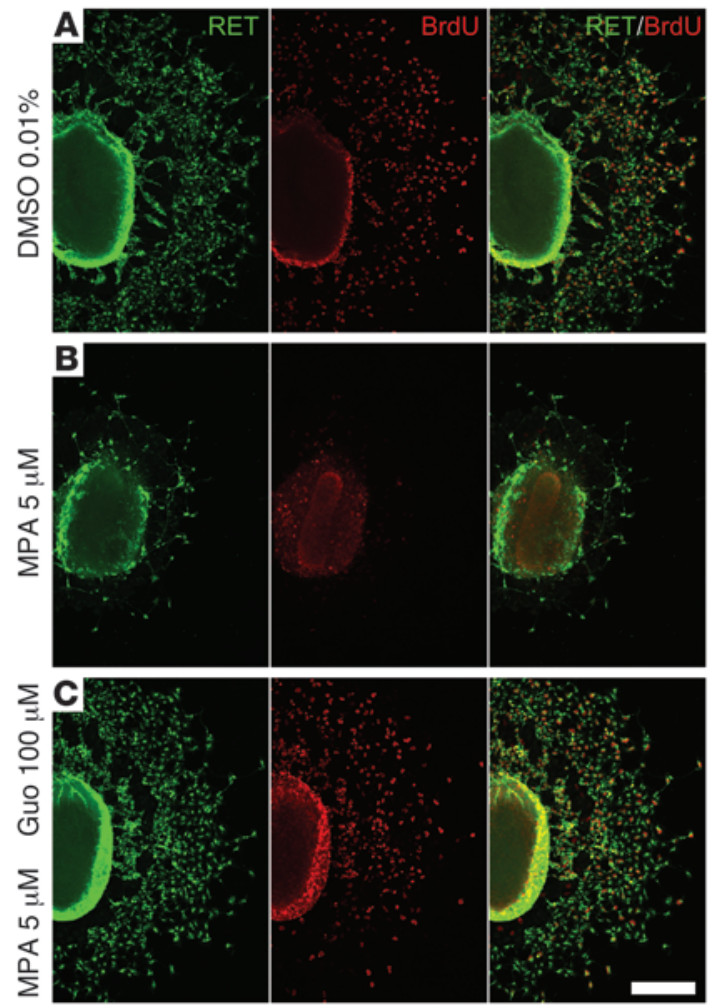

$\mathbf{F}$

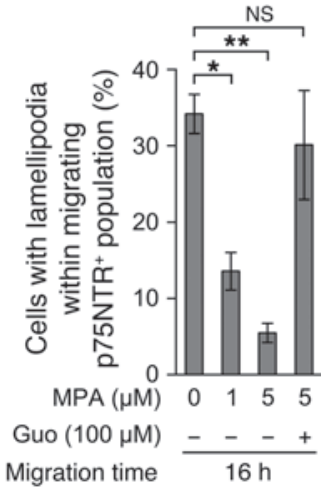

D

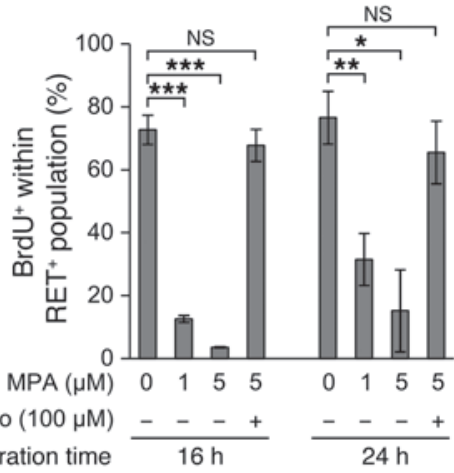

E

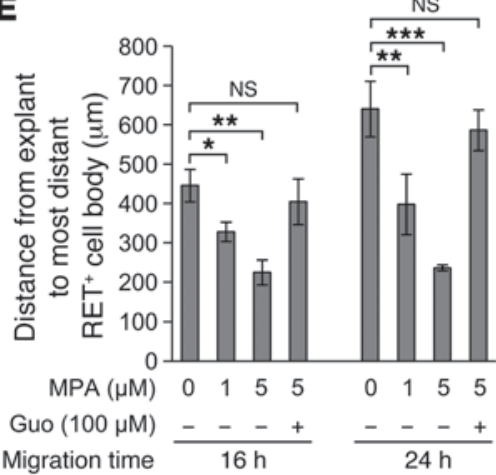

I

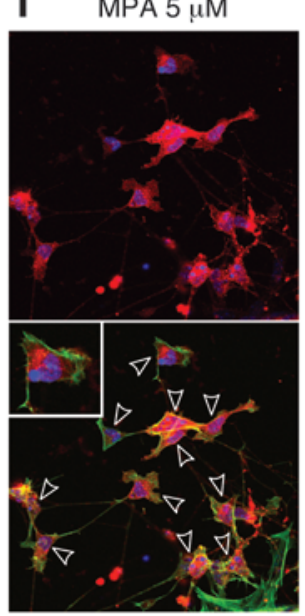

MPA $5 \mu \mathrm{M}$ J Guo $100 \mu \mathrm{M}$

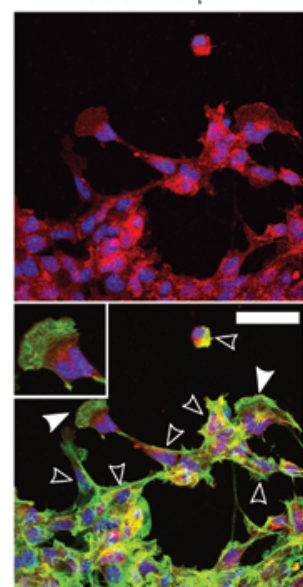

Figure 2

MPA reduced ENCDC migration, DNA synthesis, and lamellipodia in explant cultures. (A-C) Low-magnification confocal micrographs of 24-hour E12.5 midgut explant cultures immunostained for RET and BrdU (explant at left of each image). Guanosine (Guo; C) completely reversed the proliferation and migration reduction caused by MPA (B). (D) Quantification of BrdU labeling index and (E) distance migrated by the RETexpressing population after 16 and 24 hours in culture. (F) MPA reduced the percentage of cells with lamellipodia within the neural crest-derived cell population (stained with anti-p75NTR) most distant from the explant, an effect that was also reversed by guanosine. (G-J) Optical sections of p75NTR- and phalloidin-stained ENCDCs demonstrated the changes in cell shape associated with MPA treatment. Filled and open arrowheads denote ENCDCs with and without lamellipodium, respectively. Insets show details of ENCDCs at the leading edge (enlarged $\times 1.8)$. Scale bars: $250 \mu \mathrm{m}(\mathbf{A}-\mathbf{C}) ; 50 \mu \mathrm{m}(\mathbf{G}-\mathbf{J}) .{ }^{\star} P<0.05,{ }^{* *} P<0.01,{ }^{* \star} P<0.001$, repeated-measures ANOVA (D and E); ANOVA (F).

depletion. Leveraging this system to determine which GTP depletion effects are cell autonomous requires a cellular marker indicating which $\mathrm{X}$ chromosome is active. To achieve this, we mated male mice carrying an X-linked EGFP transgene (30) to $\mathrm{Hprt}^{+/}$- females, and E12.5 midgut explants were cultured with $5 \mu \mathrm{M}$ MPA and
$100 \mu \mathrm{M}$ guanosine, conditions that completely rescue MPA effects in WT explants. This mating resulted in 4 possible genotypes (Figure 4A). Of these, the female $\mathrm{X}-\mathrm{EGFP}^{+}, \mathrm{Hprt}^{+/}$genotype produces an embryo containing a mixture of cells with normal HPRT activity $\left(\mathrm{EGFP}^{+}\right)$and EGFP- cells with no HPRT activity. Therefore, each 

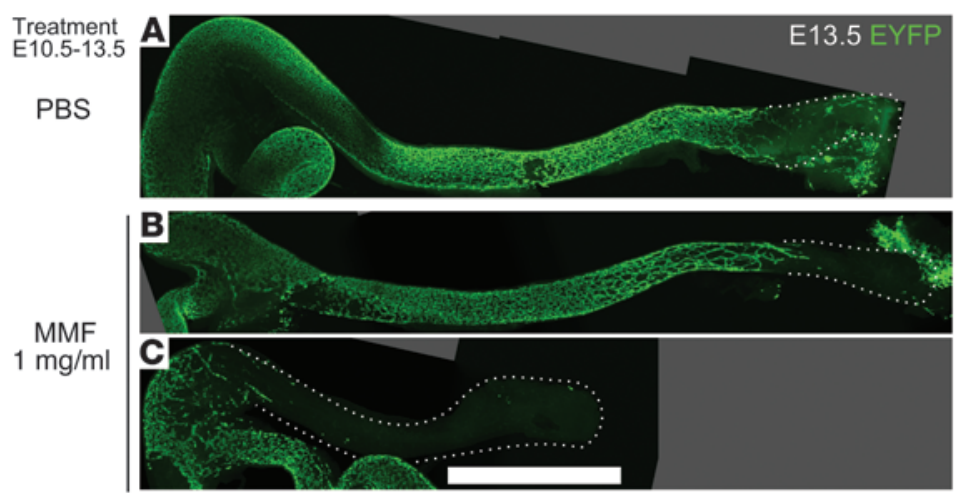

EYFP

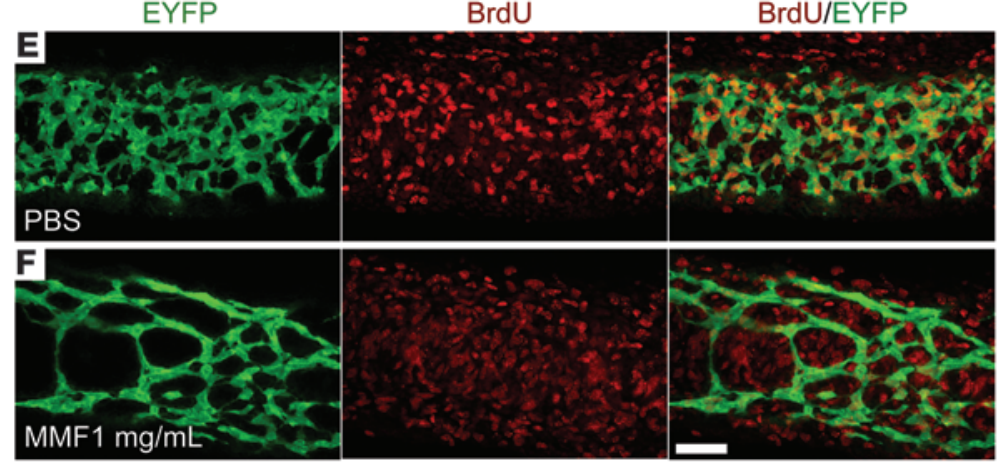

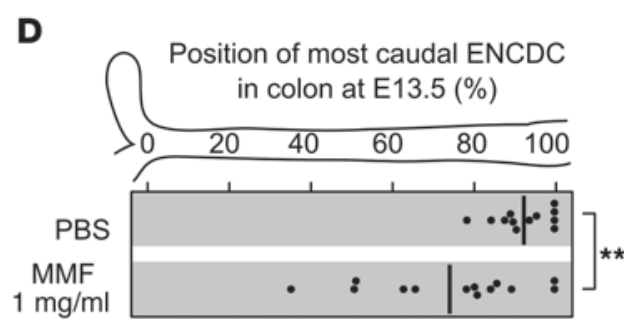
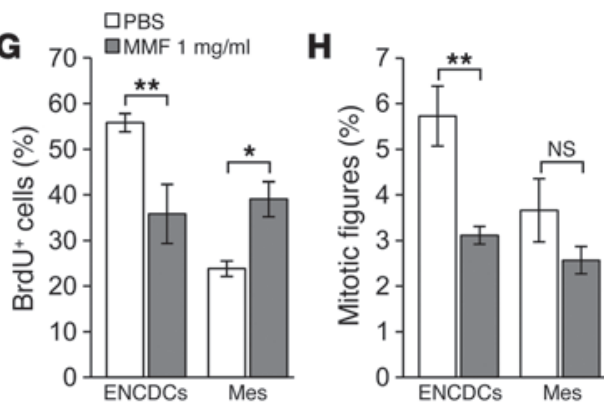

Figure 3

MMF treatment reduces ENCDC migration and DNA synthesis in vivo. (A-D) Oral treatment of pregnant B6 dams with MMF from E10.5 to E13.5 reduced the colonization of the hindgut at E13.5. Shown are stitched maximum-intensity projections of untreated $(\mathbf{A})$, mildly affected (B), and severely affected (C) fetal colons with EYFP-marked ENCDCs, demonstrating MMF's inhibitory effect on ENCDC wavefront migration in vivo. Dotted lines denote outline of bowel. (D) The position within each E13.5 colon of the most caudal ENCDC cell body (ascertained by EYFP or by SOX10 staining in EYFP- littermates) is plotted for each treatment (thick lines denote mean). (E and F) 8- $\mu$ m-thick maximumintensity projections of EYFP- and BrdU-labeled E13.5 colons. (G) Counting of BrdU+ cells within the volumes in $\mathbf{F}$ demonstrated a reduced proportion of BrdU+ ENCDCs and an increased proportion of BrdU+ mesenchymal cells after MMF treatment. mes, non-ENCDC mesenchyme. $(\mathbf{H})$ Counting of mitotic figures showed that the proportion of ENCDCs undergoing mitosis was reduced, while the mitotic index of the mesenchyme was not significantly changed. Scale bars: $1 \mathrm{~mm}(\mathbf{A}-\mathbf{C}) ; 50 \mu \mathrm{m}(\mathbf{E}$ and $\mathbf{F}) .{ }^{\star} P<0.05,{ }^{\star \star} P<0.01$, Student's $t$ test (D); ANOVA (G); ANOVA on log-transformed values $(\mathbf{H})$.

explant from X-EGFP ${ }^{+}, \mathrm{Hprt}^{+/-}$fetuses contains a mixture of guanosine-rescuable ENCDCs $\left(\mathrm{EGFP}^{+}\right)$and $\mathrm{EGFP}^{-}$cells that cannot convert guanosine to GMP, GTP, or dGTP. The other 3 genotypes served as controls. As expected, MPA- and guanosine-treated male $\left(\right.$ Hprt $\left.^{/ Y}\right)$ explants had much less migration, less BrdU incorporation, and fewer lamellipodia than WT explants (Figure 4, D, F, and $\mathrm{H}$ ), which confirmed that HPRT is required for guanosine rescue. In female WT explants, ENCDCs incorporated BrdU equally within $\mathrm{EGFP}^{+}$and $\mathrm{EGFP}^{-}$populations, as expected, whereas EGFP- cells in female X-EGFP ${ }^{+}, \mathrm{Hprt}^{+/-}$cultures incorporated very little BrdU compared with neighboring $\mathrm{EGFP}^{+}$cells (Figure 4, B, C, and E). These observations confirmed that DNA synthesis is cell autonomous with respect to GTP depletion and that guanine nucleotides are inefficiently transferred from rescued WT to mutant cells.

Unexpectedly, the overall migration from $\mathrm{Hprt}^{+/-}$explants was equivalent to WT levels (Figure 4F). Moreover, despite absent BrdU incorporation, within $H p r t^{+/}$cultures, HPRT-deficient ENCDCs $\left(\mathrm{EGFP}^{-}\right)$migrated indistinguishably from neighboring HPRTexpressing $\mathrm{EGFP}^{+}$cells (Figure 4G). Furthermore, consistent with migration competence, the proportion of EGFP- ENCDCs with lamellipodia in $\mathrm{Hprt}^{+/}$cultures was not reduced (Figure 4I).
Thus, while DNA synthesis, cell migration, and lamellipodia were all affected by GTP depletion and required HPRT for guanosine rescue, only effects on DNA synthesis were cell autonomous with respect to GTP pools. ENCDCs with adequate GTP fully rescued the ability of adjacent GTP-depleted ENCDCs to migrate in culture. Consistent with these results, time-lapse imaging of isolated ENCDCs confirmed that MPA did not reduce cell motility. However, in explant cultures where dense clusters of ENCDCs migrate outward, time-lapse imaging confirmed slower migration of MPAtreated ENCDCs (Supplemental Figure 8). Because ENCDC proliferation also drives migration in vivo (27), these data suggest that the primary defect that causes HSCR-like delays in ENCDC migration after MPA/MMF treatment is reduced cell proliferation that secondarily reduces ENCDC colonization of distal bowel.

$M M F$ increased penetrance and extent of aganglionosis. MPA is teratogenic (31) in humans, and some MPA-associated malformations are plausibly due to defective neural crest-derived cell development (32). However, aganglionosis has not been described in MPA-exposed children or animals. We hypothesized that MPA's effects on perinatal ENS structure might be more dramatic when combined with predisposing but incompletely penetrant muta- 
A

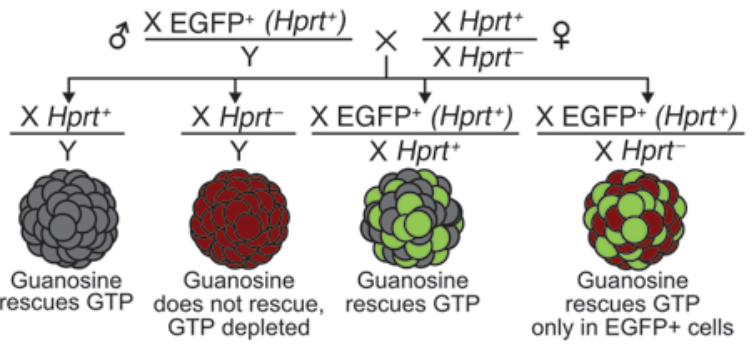

HPRT expressing cell HPRT deficient cell
HPRT expressing, EGFP+ cell
E

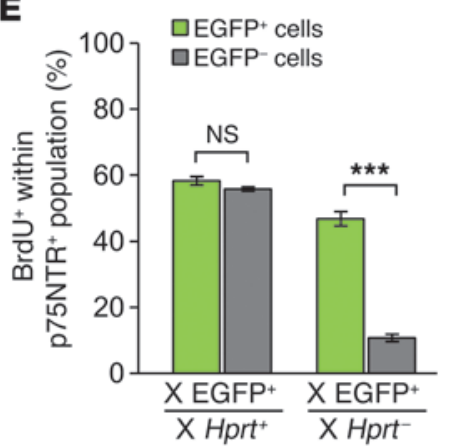

F

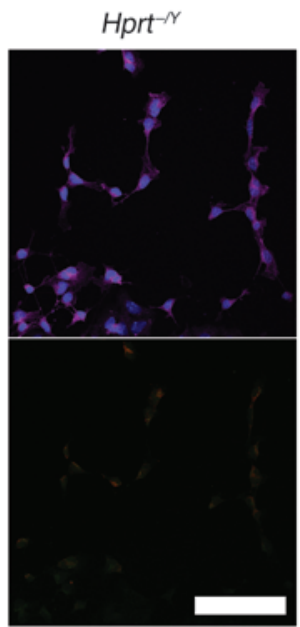

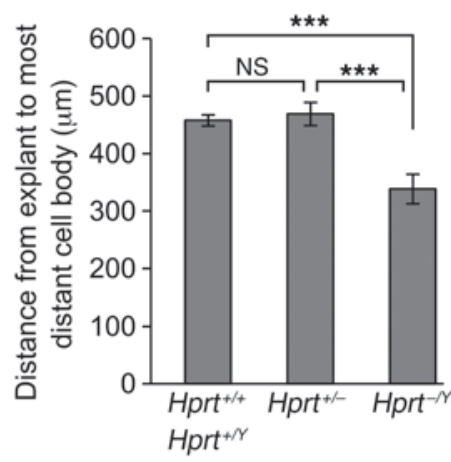

C $\frac{\mathrm{XEGFP}+}{\mathrm{X} \mathrm{Hprt}}$

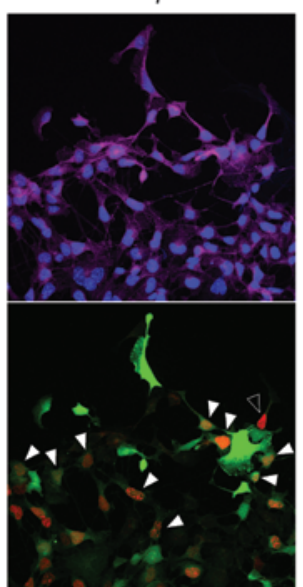

G

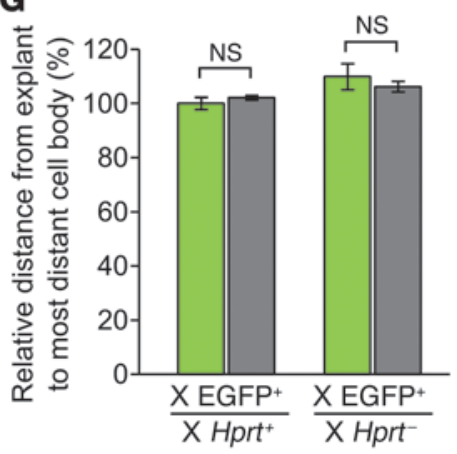

H

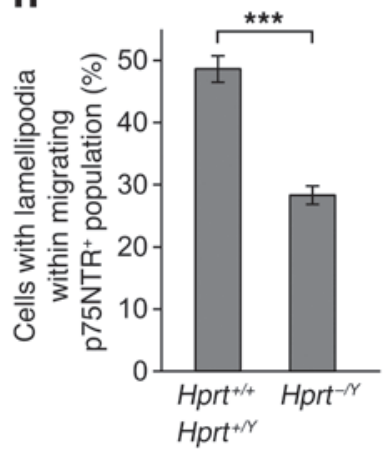

I

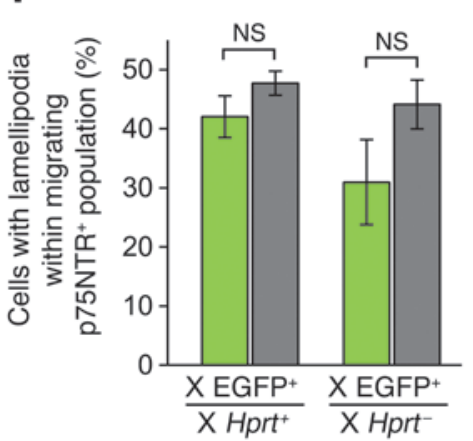

Figure 4

Mosaic analysis reveals that effects of GTP depletion on migration and lamellipodia are non-cell autonomous. (A) Mating scheme, genotypes, HPRT and EGFP expression patterns, and GTP depletion status of each population when cultured in the presence of both MPA and guanosine. These conditions create mixed cultures of GTP-depleted and EGFP-marked, guanosine-rescued ENCDCs in X-EGFP+, $\mathrm{Hprt}^{+/-}$explants, allowing migration of individual GTP-depleted ENCDCs to be examined in the context of a field of rescued ENCDCs. (B-D) BrdU labeling revealed that guanosine rescued DNA synthesis in all ENCDCs in X-EGFP+, Hprt+/+ explants (B), but only rescued DNA synthesis within the HPRT-expressing ENCDCs marked by EGFP in X-EGFP+, Hprt+/- explants (C). As expected, guanosine failed to rescue DNA synthesis in Hprt ${ }^{-1 /}$ ENCDCs (D). Filled arrowheads denote BrdU+EGFP+ double-positive ENCDCs; open arrowheads denote BrdU+GFP- ENCDCs. (E) Quantification of BrdU labeling in mosaic explants. (F) ENCDC migration out of explants was impaired in $\mathrm{Hprt}^{/ /}$explants, as expected, but $\mathrm{Hprt}^{+/+}$and $\mathrm{Hprt}^{+/-}$explants produced similar ENCDC migration distances. (G) Quantification of migration within the depleted (EGFP-) and rescued (EGFP+) populations of female $\mathrm{Hprt}^{+/-}$cells demonstrated that GTP-depleted cells did not migrate any less efficiently than rescued cells when surrounded by rescued cells. Similarly, while lamellipodia were reduced in $\mathrm{Hprt}^{/ \mathrm{Y}}$ explants $(\mathbf{H})$, they were not reduced within the GTP-depleted ENCDC population in $\mathrm{Hprt}^{+/-}$explant cultures (I). Scale bar: $50 \mu \mathrm{m}(\mathbf{B}-\mathbf{D})$. ${ }^{* \star} P<0.001$, paired $t$ test (E and I); ANOVA (F); Wilcoxon signed-rank test (G); $t$ test (H). 
A

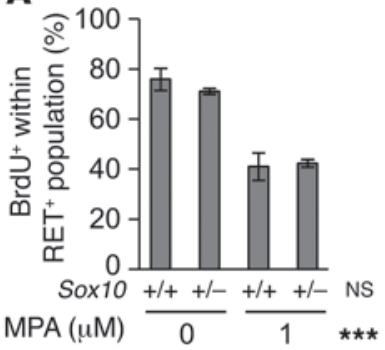

B

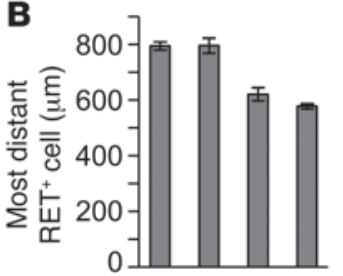

Sox10 +/+ +/- +/+ +/- NS $\operatorname{MPA}(\mu \mathrm{M}) \overline{0} \frac{}{1}$ **

D

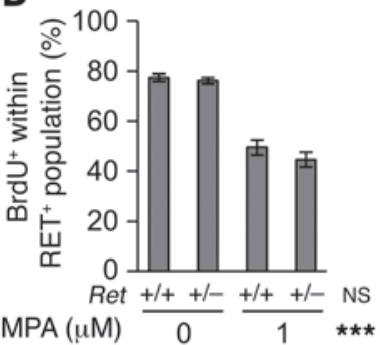

E

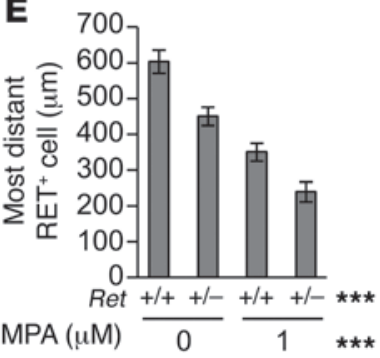

C

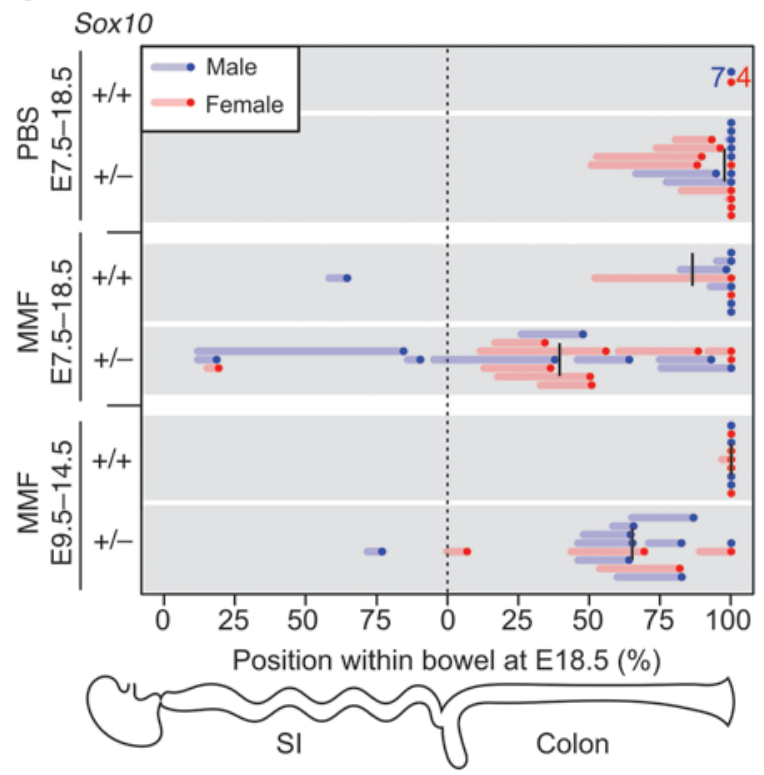

$\mathbf{F}$

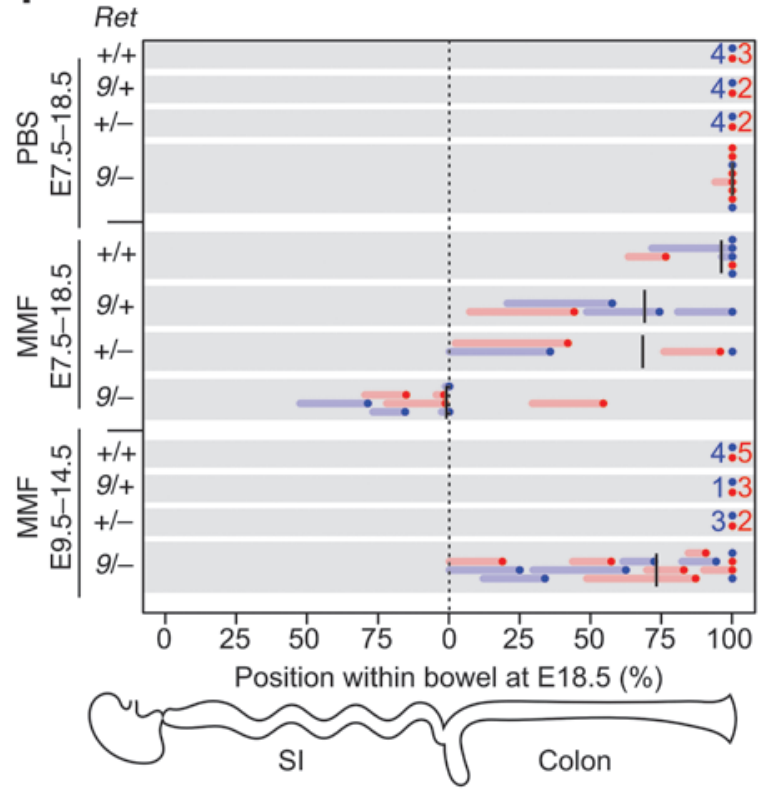

Figure 5

MMF treatment interacts with Ret and Sox10 mutations to increase penetrance and severity of HSCR-like pathology. (A and D) MPA treatment in 24-hour explant cultures revealed that neither Ret nor Sox 10 heterozygosity affected BrdU incorporation. (B and E) In contrast, Ret but not Sox10 heterozygosity reduced ENCDC migration distance, and MPA treatment had an additive effect on ENCDC migration distance. Interaction terms were not statistically significant. (C and F) Pregnant dams were provided MMF or control PBS in drinking water, and the ENS was examined at E18.5 with neuronal fiber (TuJ1) and soma (HuC/HuD) markers. The position of the most caudal soma within the intestine is plotted as a dot, and the region of hypoganglionosis is plotted as a line. Mean positions of aganglionosis are denoted by black lines. Groups without abnormal fetuses are summarized as 1 dot and number. Treatment with MMF from E7.5 to E18.5 resulted in hypoganglionosis and aganglionosis with genotype-dependant penetrance and severity. Treatment with MMF from E9.5 to E14.5 demonstrated genotypedependent reversal of the MMF-induced developmental delays. ${ }^{* \star *} P<0.001$, 2-way ANOVA (A, B, D, and E). tions, and that HSCR might only be induced by MPA when such mutations are present. We therefore exposed the developing ENS of Sox $10^{\mathrm{LacZ} /+}$ mice to MPA. Sox $10^{\mathrm{LacZ} /+}$ mice have partially penetrant aganglionosis and hypoganglionosis (33), modeling highpenetrance HSCR in Waardenburg syndrome type IV. Explant cultures of Sox $10^{\text {LacZ/+ }}$ E12.5 bowel had ENCDC migration and BrdU labeling that were equivalent to WT cultures under control conditions. Importantly, MPA had similar effects on WT and Sox10 LacZ/+ ENCDCs (Figure 5, A and B). Thus, in this short-term culture, MPA was not more toxic to mutant than to WT ENCDCs.

Next, we examined the interaction between MMF and Sox10 in vivo. Since Sox10 is haploinsufficient in the developing ENS, we crossed Sox $10^{\mathrm{LacZ} /{ }^{+}}$males with B6 females (34) and treated them with MMF throughout prenatal ENS development (E7.5-E18.5). We attempted postnatal evaluation, but some MMF-treated pups had exencephaly and died immediately after birth. To avoid missing more severely affected animals, we harvested E18.5 fetuses. Occasionally, control dams delivered at E18.5, but ENS structure was similar to fetuses that had not delivered. Whole-mount immunohistochemistry for neuronal processes (TuJ1) and somata (HuC/HuD) demonstrated that Sox10 mutations and MMF individually caused partially penetrant colonic hypoganglionosis and aganglionosis (Figure 5C). The combination of Sox10 mutation and MMF resulted in extensive bowel aganglionosis.

We next tested MPA on Ret $^{+/-}$ENCDCs in culture, since RET is mutated in most human HSCR cases (2). Ret $^{+/-}$mice, however, are never aganglionic or hypoganglionic (35). In contrast to Sox $10^{\text {LacZ/++ }}$ ENCDCs, Ret $^{+/-}$ENCDCs did not migrate as efficiently as WT in culture, although BrdU labeling was unaffected by Ret genotype (Figure 5, D and E). MPA and Ret heterozygosity had additive effects on 

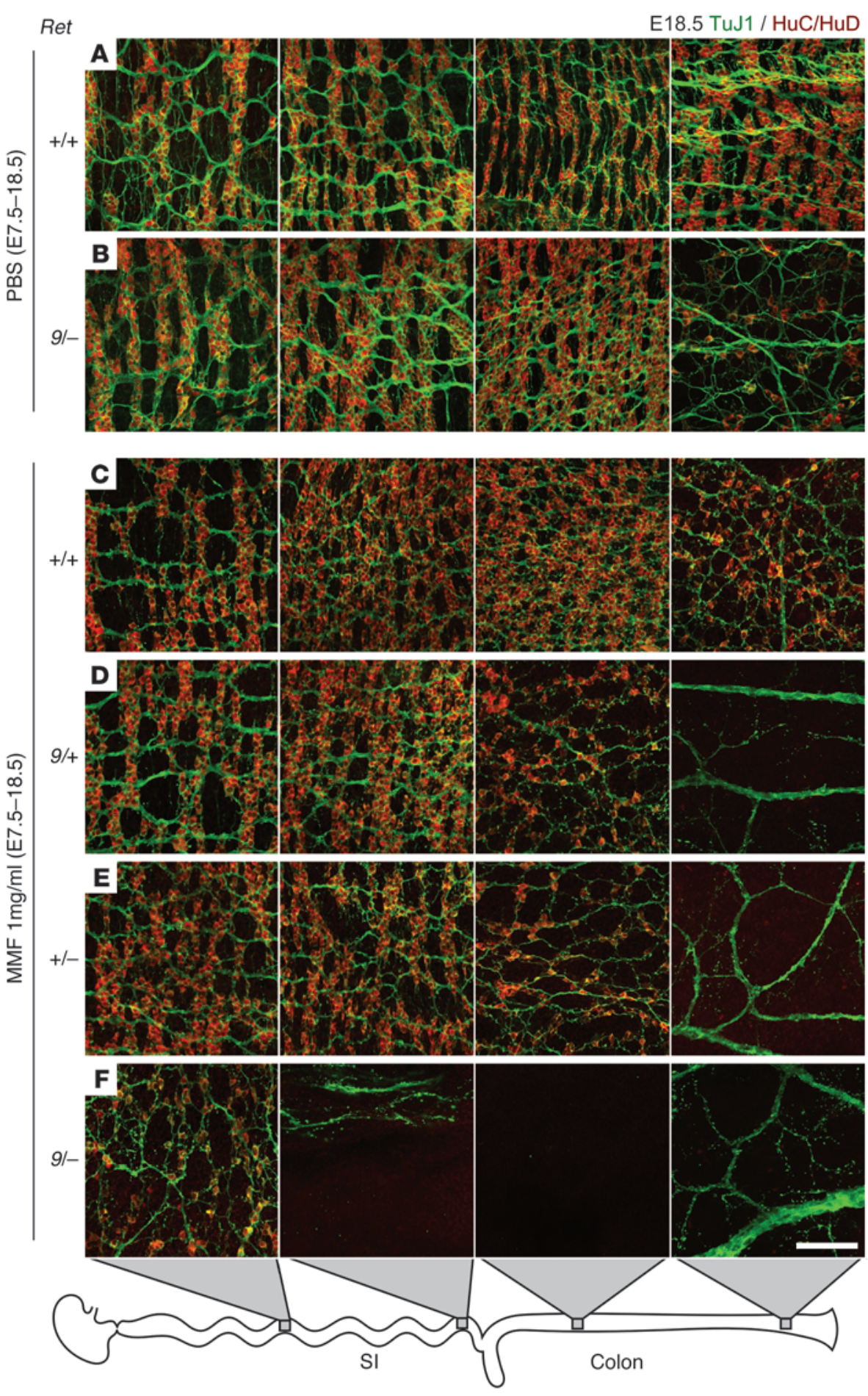

\section{Figure 6}

Representative maximum-intensity projections of myenteric plexus in the mid-small bowel, terminal ileum, proximal colon, and terminal colon from PBS-exposed WT (A) and $\operatorname{Ret}^{9 /-}$ (B) fetuses and MMF-treated $\operatorname{Ret}^{+/+}(\mathbf{C}), \operatorname{Ret}^{9 /+}(\mathbf{D}), \operatorname{Ret}^{+/-}(\mathbf{E})$, and $\operatorname{Ret}^{9 /-}$ (F) genotypes. PBS-exposed Ret $^{9 /-}$ colon (B) displayed distal hypoganglionosis. (D-F) TuJ1 staining demonstrated thick nerve bundles in the aganglionic terminal colons and disorganized fibers in hypoganglionic regions. Scale bar: $100 \mu \mathrm{m}(\mathbf{A}-\mathbf{F})$.

even without MMF (26), but found no aganglionosis and only 1 mouse with obvious hypoganglionosis (Figure 5F and Figure 6B). Other genotypes did not have detectable ENS abnormalities in control fetuses (Figure 5F and Figure 6A). In contrast, 2 of 6 MMF-treated $\mathrm{Ret}^{+/+}$fetuses had hypoganglionic or aganglionic colons (Figure 5F and Figure 6C). Furthermore, MMF-treated Ret ${ }^{9 /+}$ and $\mathrm{Ret}^{+/-}$fetuses had longer regions of aganglionic colon than WT fetuses (Figure 5F and Figure 6, D and E), demonstrating a synergistic effect. Strikingly, all 8 MMF-treated Ret $^{9 /-}$ fetuses had aganglionosis, often extending into small bowel (Figure 5F and Figure $6 \mathrm{~F}$ ). These findings are remarkable since the Ret ${ }^{9 /-}$ genotype closely mimics heterozygous RET mutations that underlie more than $25 \%$ of human HSCR. Unlike human HSCR, however, sex did not affect the penetrance or the extent of ENS abnormalities in MMFtreated Ret mutant mice.

Thus, MMF treatment from E7.5 onward caused Hirschsprung-like aganglionosis that was significantly worse in genetically susceptible mice. We also tested the hypothesis that a more limited period of MMF exposure might cause permanent distal bowel aganglionosis. ENCDCs normally first enter the bowel at E9.5 (39) and reach the end of the colon by E13.5, but the colon becomes less permissive $(40,41)$ to ENCDC migration at E14.5. To determine

distance migrated, consistent with known roles for RET in ENCDC migration $(11,36)$ and MPA's primary effect on proliferation.

Since heterozygous null Ret mutations do not delay ENCDC colonization of fetal bowel (35) and only slightly alter adult ENS structure (37), we used a hypomorphic allele, $\operatorname{Ret}^{9}$, that causes partially penetrant aganglionosis (38) to test the effects of MMF in vivo. Ret $^{9 /+}$ males were bred to Ret $^{+/-}$females on a B6 background, and pregnant dams were treated with MMF from E7.5 to E18.5. We expected $\operatorname{Ret}^{9 /-}$ fetuses to have partially penetrant aganglionosis whether MMF treatment during the critical period of ENCDC migration causes permanent or transient distal bowel aganglionosis, we switched dams carrying Sox10 and Ret litters from PBS to MMF for the interval from E9.5 to E14.5 and then allowed them to recover on PBS from E14.5 to E18.5. For Sox10 and Ret matings (Figure 5, C and F), the colons of all WT fetuses and intermediate Ret genotype fetuses were completely colonized at E18.5, although 1 Sox $10^{+/+}$fetus had a hypoganglionic terminal colon. In contrast, aganglionosis was highly - although not universally - 
penetrant in Ret $^{9 /-}$ fetuses treated with MMF from E9.5 to E14.5 and was confined to the colon (Figure 5F). Furthermore, although the penetrance of aganglionosis in Sox $10^{\mathrm{Lac} /+}$ fetuses treated with MMF from E9.5 to E18.5 was very similar to those treated from E7.5 to E18.5, the aganglionic segments were shorter on average if mice did not receive MMF before E9.5 and after E14.5. Collectively, these data suggest that significant ENCDC migration delays may be reversible, but the most susceptible genotypes lack the capacity to recover from transient MMF exposure.

It is important to note that the preceding data described colonization of the bowel by ENCDCs derived from the vagal region of the neural crest. Interestingly, in mice with aganglionic bowel, the terminal third of the colon often had single neurons or isolated clumps of neurons ( $<150$ neurons per colon) associated with extrinsic nerve bundles and separated from vagal ENCDCs by long segments of bowel that were completely devoid of enteric neurons. These rare neurons are likely to arise from ENCDCs derived from the sacral region of the neural crest.

In addition to ENS defects, MMF-treated mice were small and had reduced bowel length (Supplemental Figure 9), exencephaly, and congenital heart defects. MMF-induced exencephaly was unaffected by genotype, but was only present in litters treated with MMF from E7.5 to E18.5 (Supplemental Table 2). Most heart defects occurred in Sox10 or Ret mutant fetuses in the E7.5-E18.5 treatment group. Although the number of fetuses was small, the infrequent heart defects in MMF-treated control mice raises the possibility that Sox10 or Ret may have unappreciated roles in cardiac crest development that are only demonstrable with additional genetic or environmental insults. We also observed $6 \mathrm{MMF}-$ exposed fetuses with orofacial clefting and 3 with iris colobomas. These defects are similar to those previously reported in MMFexposed human infants (31).

\section{Discussion}

HSCR and other problems with ENS development are known to occur in individuals with many well-established genetic defects (2), which suggests that these disorders are not preventable. The gene defects and chromosomal anomalies that predispose to HSCR, however, are all partially penetrant and cause variable degrees of aganglionosis. This variation in phenotype in individuals who share the same underlying primary genetic defect is thought to occur, at least in part, because of interactions among genes needed for normal development. Our current data provide the first direct evidence that specific medicines affect ENS development, causing distal bowel aganglionosis in mice and fish that mimics human HSCR. In addition, our data showed dramatic gene-environment interactions. Importantly, these studies suggest the possibility that any factor that reduces ENCDC proliferation might increase the risk of distal bowel aganglionosis and that some cases of HSCR might be prevented by careful optimization of nongenetic risk factors during early pregnancy.

Most compounds found to inhibit distal bowel colonization by ENCDCs in fish lack obvious links to previously recognized ENS developmental pathways. Furthermore, because we tested only 1 drug concentration, many additional medications are probably detrimental to the developing ENS. Besides MPA, 2 additional medications deserve comment. Lovastatin (Mevinolin; Merck) is a commonly used inhibitor of the rate-limiting step in de novo cholesterol biosynthesis (HMG-CoA reductase), which is interesting because DHCR7 mutations disrupt the final step in cholesterol biosynthesis, causing HSCR as a component of Smith-Lemli-Opitz syndrome (42). Artesunate is a common malaria treatment that may increase ROS, and in Tcof1 mutant mice, additional oxidative stress delays ENCDC migration (43). These findings highlight the many complex pathways needed for ENCDC colonization of fetal bowel and the potential for diverse medicine classes to increase HSCR occurrence. This is especially important since families with 1 child with HSCR have a 50- to 1,600-fold increased risk of having another child with the same life-threatening disease (2).

We investigated the immunosuppressant MPA in more detail, because it profoundly inhibited zebrafish ENS development at concentrations in the low human therapeutic range and represents the only drug identified associated with specific patterns of human birth defects (31). Despite inhibiting the ubiquitous process of GMP biosynthesis, some MPA-associated defects (e.g., cardiac defects, craniofacial defects, and coloboma) suggest that IMPDH inhibition disproportionately affects neural crest-derived cells (32). Interestingly, Drosophila IMPDH mutations (raspberry) cause mistargeting of photoreceptor axons (44), and MPA impairs cranial nerve development in rat embryos (45), demonstrating that defects in other neural cell types can be caused by abnormal purine metabolism. We are not aware of human ENS malformations reported after MPA or MMF exposure, but HSCR occurrence after exposure likely requires predisposing gene mutations. Here we showed that MPA caused dose-dependent distal ENS malformations in fish and mice, that MPA impaired ENCDC migration by depleting guanine nucleotides and reducing proliferation, and that mutations that model HSCR predisposition dramatically increased MMF's teratogenic effects on distal bowel colonization by ENCDCs.

The unique way in which the ENS develops may explain why ENCDCs are particularly sensitive to MPA/MMF. One possibility is that the relatively high rate of ENCDC proliferation compared with that of neighboring mesenchymal cells requires ENCDCs to synthesize guanine nucleotides more rapidly via IMPDH and the de novo synthesis pathway. The elevated levels of IMPDH observed in ENCDCs compared with adjacent cells are consistent with this hypothesis, as are the different effects of MMF in different cell types. As expected, MPA/MMF dramatically reduced BrdU incorporation and mitotic figures in ENCDCs. Remarkably, the effects of MPA/MMF in neighboring mesenchyme were more complex. In contrast to ENCDCs, a higher proportion of mesenchymal cells incorporated BrdU after MPA/MMF treatment, but without a concomitant increase in mitoses. This might occur if MMF-treated mesenchymal cells have enough guanine nucleotide to enter S-phase, but have a prolonged period of DNA synthesis because of limited dGTP availability. Alternatively, guanine nucleotide depletion could cause DNA damage, and the elevated levels of BrdU incorporation reflect DNA repair. In any case, the effect on mammalian development of blocking a ubiquitous enzyme like IMPDH depends not only on how the drug affects the biology of individual cells, but also on how important that perturbation is for particular aspects of development. For example, proliferation is essential for ENCDCs to efficiently colonize the distal bowel (27). Reduced proliferation of other cell types might lead to smaller organs, but might not cause a structural defect, because proliferation is not essential for building that structure during the interval of drug exposure.

For our present studies, it was important to determine whether distal bowel aganglionosis in MPA/MMF-treated mice occurred because of reduced ENCDC proliferation (27) or whether deple- 
tion of guanine nucleotides directly affected proteins needed for cell migration. In mesangial (46) and endothelial (47) cells, for example, MPA-mediated GTP depletion led to reduced levels of active (GTP-bound) Rho-family GTPases, including RAC1, a key regulator of the actin cytoskeleton. Our experiments, however, strongly suggested that MPA/MMF in the doses tested did not directly impair cell migration. Specifically, we found that random motility of EYFP-labeled ENCDCs was unchanged by MPA (Supplemental Figure 8) when cells were cultured at a density that reduced cell-cell contact. Furthermore, using X-inactivation mosaicism, we deleted HPRT and depleted GTP in single ENCDCs that were surrounded by "normal" guanosine-rescued ENCDCs. In these cultures, the nonrescued ENCDCs remained BrdU-, excluding cell-to-cell transfer of any significant amount of guanine nucleotide, but the GTP-depleted cells appeared to migrate essentially normally. Thus, with 2 different approaches, we demonstrated that GTP-depleted ENCDCs can move at normal speeds, but fail to migrate effectively unless they are surrounded by adjacent normally proliferating ENCDCs. This result is conceptually related to the findings that Sox 10 and Ednrb mutations have noncell-autonomous effects on ENCDC migration in aggregation chimeras $(48,49)$ and that proliferation inhibitors $(27)$ or mechanical reduction of ENCDC density (35) can reduce ENCDC colonization of cultured bowel. The molecular mechanisms that allow ENCDCs to sense and react to local ENCDC density or that control the unpredictable trajectories of individual ENCDCs remain unknown, although in other neural crest cells, both diffusible (50) and contact-mediated (51) signals are involved.

We therefore propose that reduced ENCDC proliferation parsimoniously explains all other observed effects of MPA on the developing ENS. These results suggest the possibility that any condition that reduces ENCDC proliferation could increase the risk of HSCR. In addition to the risk of bowel aganglionosis in WT mice after MPA/MMF treatment, our data showed dramatic gene-environment interactions between MMF and either Sox10 or Ret mutations at term. MMF caused distal bowel aganglionosis when administered for the entire period of prenatal neural crest development (E7.5-E18.5) or during the period of ENCDC migration (E9.5-E14.5). Interestingly, ENCDCs were able to recover from a transient MMF-induced developmental delay in WT mice and in mice with "mild" Ret genotypes, but not in Sox $10^{\text {LacZ/+ }}$ or $R e t^{9 /-}$ fetuses. Furthermore, MPA/MMF appeared to inhibit bowel colonization by both vagal and sacral neural crests. The sacral neural crest normally migrates proximally through terminal colon and forms $10 \%-20 \%$ of the distal colonic ENS (52). Although we observed isolated neurons in each aganglionic colon, sacral ENCDC derivatives, if present, are greatly diminished in number by MMF and/or the genetic lesions evaluated (i.e., less than $2 \%$ of anticipated sacral-derived neurons in this region). If the same phenomena occur in humans, then many potentially detrimental drug exposures will only cause HSCR in children with underlying predisposing mutations.

A few interesting differences were noted between in vivo versus explant culture results for Ret and Sox10 mutant animals. In vivo, $\mathrm{Ret}^{+/+}$and $\mathrm{Ret}^{+/-}$ENCDCs migrate at the same rate through the colon (35), but we noted reduced migration of Ret $^{+/-}$ENCDCs from cultured gut explants compared with WT cells. This may occur because the supraphysiologic level of GDNF used in culture saturates all RET receptors, whereas in vivo GDNF is limiting even in $\mathrm{Ret}^{+/-}$mice (37). In contrast, Sox10 mutant and WT ENCDCs migrated an equal distance from gut explants in vitro, while the same Sox10 mutation impaired distal bowel colonization in vivo. This illustrates the importance of coupling in vitro and in vivo studies, since some defects will not be detected in the culture system currently in use. Differences that might be required to detect Sox10mediated defects include the much longer ENCDC migration time in vivo; the diverse set of factors affecting stem cell renewal, differentiation, and migration; and the 3-dimensional environment in vivo that requires degradation of the extracellular matrix (53) and may engage alternate integrin or adherence protein signaling.

Collectively, our results provide the first strong evidence that medicines may affect ENS development and that potent gene-environment interactions dramatically alter the risk of Hirschsprunglike disease. Combined with our previous demonstration that vitamin A deficiency increases Hirschsprung-like defects in concert with Ret mutations (12), the present work adds credibility to the hypothesis that unappreciated maternal nongenetic factors influence HSCR risk. Oral MMF may also be a valuable experimental tool to probe for genetic factors that predispose to ENS abnormalities, but are not severe enough to cause disease in the absence of additional defects.

Finally, these studies demonstrated a profound linkage among basic processes in cell metabolism and specific mammalian ENS developmental defects. This work supports the idea that any stressor that reduces the efficiency of ENCDC proliferation might increase HSCR occurrence and severity in children with predisposing mutations, even without altering "classical" ENS developmental pathways. De novo purine biosynthesis, for example, is a multistep process for which enzymes require nicotinamide, folate, and vitamin B12. Focused investigation of clinically relevant antimetabolic stressors such as folate and B12 deficiency, antifolate medicines (e.g., trimethoprim and methotrexate), and other antimetabolites (e.g., azathioprine and 6-mercaptopurine) may identify immediate candidate interventions for reducing the incidence and severity of HSCR in genetically predisposed children. In parallel with work in model systems, these studies suggest that human case-control epidemiologic investigation is appropriate and may uncover avoidable maternal exposures or health conditions that could reduce the risk of dangerous neural crest-dependent birth defects like HSCR.

\section{Methods}

Zebrafish. WT (AB) in vitro-fertilized embryos were treated $(n \geq 6$ embryos per drug) with $10 \mu \mathrm{M}$ drug and 1\% DMSO in E3 Screening Media (54) from 34 to $96 \mathrm{hpf}$. 1\% DMSO did not induce any defects, but may increase sensitivity, as $2 \%$ DMSO alone inhibited ENCDC migration. Distance from the most-caudal neuron $\left(\mathrm{HuC} / \mathrm{HuD}^{+}\right)$to the bowel terminus was measured ( $n>9,000$ larvae) using a micrometer-calibrated eyepiece grid. Compounds were retested if mean uncolonized distance exceeded $125 \mu \mathrm{m}$, results were consistent between experiments, and the compound might cross the placenta in mammals. Drugs causing death at $10 \mu \mathrm{M}$ were retested at lower concentrations. Larvae with $>100 \mu \mathrm{m}$ of uncolonized bowel were considered to be affected for the $\mathrm{TD}_{50}$ calculations.

Mice. Vaginal plug day was considered E0.5. CF1 mice were from Charles River. B6 (C57BL/6J), C3Fe (C3HeBFe/J), 129X1 (129X1Sv/J), Wnt1-Cre

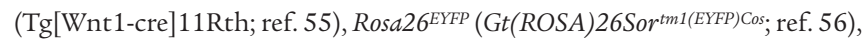
and X-EGFP ${ }^{+}$(Tg[CAG-EGFP]D4Nagy/J; ref. 30) mice were from the Jackson Laboratory. Other mouse strains and genetic backgrounds used were $\operatorname{Ret}^{T G M}$ (null allele Ret ${ }^{\text {tmIIJmi} ; ~ r e f . ~ 57) ~ o n ~ a ~ B 6 ~ b a c k g r o u n d, ~ S o x 10 ~}{ }^{\text {LacZ }}$ (null allele

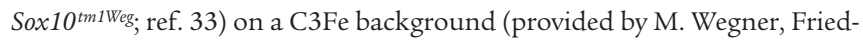


rich Alexander University Erlangen Nuremberg, Erlangen, Germany, and M. Southard-Smith, Vanderbilt University, Nashville, Tennessee, USA), and Ret $^{9}$ (hypomorphic allele Ret ${ }^{\operatorname{tm} 2(R E T) J m i}$; provided by S. Jain, Washington University School of Medicine, St. Louis, Missouri, USA; ref. 38) backcrossed to 129X1 for 2-5 generations. Wnt1-Cre Rosa26 $6^{\text {EYFP/EYFP }}$ and Hprt ${ }^{-}$(Hprt ${ }^{b-m 3}$; provided by B.S. Mitchell, Stanford University, Stanford, California, USA; ref. 28) mice were on a mixed background. PCR genotyping (58) for Sox $10^{\text {LacZ }}$ and Wnt1-Cre used previously published primers (58); other primers are listed in Supplemental Table 3.

MPA and MMF treatment. Dams were injected daily with MPA (SigmaAldrich; catalog no. M3536) in DMSO or with DMSO alone $(1.24 \mu \mathrm{l} / \mathrm{g}$ body weight, 31.25-250 mM MPA, intraperitoneal) or given prodrug MMF (Accord Healthcare, NDC catalog no. 16729-094) at $1 \mathrm{mg} / \mathrm{ml}$ in $0.25 \times$ PBS adjusted to $\mathrm{pH} 3.6$ as drinking water (23). MMF and PBS groups drank equal amounts. Cardiovascular anatomy at E18.5 was visualized under a dissection stereomicroscope by left ventricle injection with India ink (Windsor Newton) diluted 1:10 in water.

Primary ENCDC culture. 300- to 500- $\mu \mathrm{m}$ slices of E12.5 small bowel were cultured on fibronectin-coated ( $250 \mu \mathrm{g} / \mathrm{ml}$; Invitrogen) Lab-Tek Permanox chamber slides (Thermo Fisher) in DMEM (high glucose), $200 \mathrm{mM}$ L-glutamine, $100 \mathrm{IU} / \mathrm{ml}$ penicillin, $100 \mathrm{~g} / \mathrm{ml}$ streptomycin, $1 \times \mathrm{B}-27$ supplement (Invitrogen), and MPA in DMSO (0.01\% final concentration). At 4 hours after plating, $50 \mathrm{ng} / \mathrm{ml}$ GDNF (59) was added to trigger migration. Cultures were maintained for an additional 16 or 24 hours $\left(37^{\circ} \mathrm{C}, 5 \% \mathrm{CO}_{2}\right)$. When appropriate, $10 \mu \mathrm{M} \mathrm{BrdU}$ was added 5 hours before fixation. All culture experiments not involving mutant mice used CF1 fetuses. Sox10, Ret, and Hprt fetuses were cultured individually and genotyped.

For measuring neurite length, E12.5 bowel was enzymatically dissociated into a single-cell suspension (22), and cells were cultured in neurobasal media supplemented with $1 \times$ B-27, $200 \mathrm{mM}$ L-glutamine, $100 \mathrm{IU} / \mathrm{ml}$ penicillin, and $100 \mathrm{~g} / \mathrm{ml}$ streptomycin. Cultures were plated at a density of 1,250 cells $/ \mathrm{cm}^{2}$ on glass chamber slides (Lab-Tek) coated with poly-D-lysine $(100 \mu \mathrm{g} / \mathrm{ml}$; Sigma-Aldrich) and laminin (BD Biosciences) and cultured for 48 hours before fixation. BrdU $(10 \mu \mathrm{M})$, DMSO or MPA $(5 \mu \mathrm{M}$ final), and GDNF $(50 \mathrm{ng} / \mathrm{ml})$ were added at plating. For time-lapse EYFP microscopy, E12.5 Wnt1-Cre Rosa26 $6^{\text {YYFP/+ }}$ midguts were cultured as dissociated cells or slices as described above and plated on fibronectin-coated glass chamber slides (Thermo Fisher). Both cells and slices were cultured in phenol red-free DMEM supplemented with B-27, L-glutamine, and antibiotics as described above. All other culture conditions were identical to those of explant cultures.

$\mathrm{X}$-inactivation mosaic analysis. $\mathrm{X}-\mathrm{EGFP}^{+}$male mice were bred to $\mathrm{Hprt}^{+/}$ females. E12.5 midgut explants resulting from these matings (X-EGFP', $\left.\mathrm{Hprt}{ }^{+-}\right)$were cultured for 16 hours in the presence of both MPA $(5 \mu \mathrm{M})$ and guanosine $(100 \mu \mathrm{M})$ to rescue all WT cells while GTP-depleting all cells that do not express HPRT. Since both Hprt and the EGFP transgene are subject to mosaic $\mathrm{X}$-inactivation, in $\mathrm{Hprt}^{+/}$- explants, EGFP marks WT (rescued) cells but not mutant (depleted) cells. EGFP expression from the transgene was weak, and visualization required immunohistochemistry.

Immunohistochemistry. Zebrafish were fixed and then stained in wholemount as previously described (19) with anti-HuC/HuD monoclonal 16A11 (250 ng/ml; Invitrogen) and Alexa Fluor 594 anti-mouse secondary (1:250; Invitrogen) antibodies before mounting in 50\% glycerol/PBS.

E18.5 mouse bowel flushed with PBS was fixed (4\% paraformaldehyde in PBS for 30 minutes at $25^{\circ} \mathrm{C}$ ), washed in PBS, and then permeabilized/ blocked for 1 hour at $25^{\circ} \mathrm{C}$ in TBST (Tris-buffered saline with $0.1 \%$ Triton-X 100), $1 \%$ cold water fish skin gelatin (Sigma-Aldrich), $100 \mathrm{mM}$ glycine, and $5 \%$ normal serum matching secondary species (Jackson ImmunoResearch). Primary antibodies (Supplemental Table 4) were incubated overnight in blocking solution at $4{ }^{\circ} \mathrm{C}$. Fluorophore-conjugated secondary antibody (Alexa Fluor 488 donkey anti-rabbit, Alexa Fluor 594 donkey anti-rabbit,
Alexa Fluor 488 donkey anti-goat, Alexa Fluor 594 donkey anti-goat, Alexa Fluor 647 goat anti-rabbit, or Alexa Fluor 647 donkey anti-goat; Invitrogen) incubation was for 1 hour at $25^{\circ} \mathrm{C}$. When appropriate, DAPI $(100 \mathrm{ng} / \mathrm{ml})$ and/or Alexa Fluor 594- or Alexa Fluor 488-conjugated phalloidin ( $4 \mathrm{U} / \mathrm{ml}$; Invitrogen) were added during secondary incubation.

An acid treatment step $\left(4 \mathrm{~N} \mathrm{HCl}, 5\right.$ minutes, $\left.25^{\circ} \mathrm{C}\right)$ was required for $\mathrm{BrdU}$ and RET staining and was performed after blocking (for TuJ1 costaining) or after antibody staining and a postfixation step (4\% paraformaldehyde in PBS for 10 minutes) for costaining of other antigens. Samples were washed twice with TBS and blocked again between acid treatment and primary antibody incubation.

E18.5 mouse bowel anti-HuC/HuD staining required an alternate procedure. Fixed samples were treated with $3 \% \mathrm{H}_{2} \mathrm{O}_{2}$ in PBS (20 minutes at room temperature) before blocking in PBST (PBS, $\mathrm{pH} 7.4$, with $1 \%$ Triton-X 100), $10 \%$ normal donkey serum, $1 \%$ cold water fish skin gelatin, and $100 \mathrm{mM}$ glycine. Endogenous biotin was blocked (Streptavidin/biotin blocking kit; Vector Labs) before an overnight $4{ }^{\circ} \mathrm{C}$ incubation with biotin-XX-conjugated anti-HuC/HuD (400 ng/ $/ \mathrm{l}$; Invitrogen; catalog no. A21272) in blocking solution. After 6 PBST washes, tissue was incubated with Alexa Fluor 594-conjugated streptavidin (1:2,000; Invitrogen) in PBST for 15 minutes at $37^{\circ} \mathrm{C}$.

qRT-PCR. Intestines (from stomach to colon) were harvested from E13.5 fetuses treated with PBS or MMF (E10.5-E13.5) and individually homogenized in $800 \mu \mathrm{l}$ TRIzOL (Invitrogen) by passage through a 26-gauge needle. RNA was isolated according to the manufacturer's instructions, with $200 \mu \mathrm{g}$ RNA-grade glycogen (Thermo Scientific) added as a carrier. $1 \mu \mathrm{g}$ of each RNA sample was treated with RQ1 DNAse (Promega) in a total volume of $10 \mu \mathrm{l}$ to degrade genomic DNA, according to the manufacturer's instructions. $1 \mu$ l of this treated RNA solution was reverse transcribed with $200 \mathrm{U}$ SuperScript II Reverse Transcriptase (Invitrogen) according to the manufacturer's instructions using $250 \mathrm{ng}$ random hexamers in a total volume of $21.1 \mu \mathrm{l}$. qPCR reactions were performed on the equivalent of $1.6 \mathrm{ng}$ of input RNA using Power SYBR Green PCR Master Mix (Applied Biosystems) and $20 \mu \mathrm{M}$ of each oligonucleotide in a $25-\mu \mathrm{l}$ reaction on a Stratagene Mx3005P thermocyler using the following cycling parameters: 10 minutes at $95^{\circ} \mathrm{C}$ followed by 40 cycles of 30 seconds at $95^{\circ} \mathrm{C}, 1$ minute at $55^{\circ} \mathrm{C}$, and 1 minute at $72^{\circ} \mathrm{C}$. Oligonucleotide sequences for Gdnf, Ece1 (60), and Gapdh (61) were described previously, and sequences for $E d n 3$ were $5^{\prime}$-TCACCAGTTATTCCGGGAGAG-3' and 5'-TAAGGCCGGTGGGCTTTATC- ${ }^{\prime}$. No-template controls and no-RT enzyme controls were performed for each primer pair. 3 replicate reactions were run for each sample-amplicon combination, and average PCR efficiencies for each amplicon were calculated using LinRegPCR software (62), which were then used to calculate efficiency-corrected fold changes (63) using Gapdh as a reference gene.

Microscopy and quantification. Micrographs were acquired with Olympus BX60 or IX71 microscopes, Axiocam CCD camera, and Axiovision software or with an Olympus FV1000 confocal microscope and Fluoview software. ImageJ was used for image processing, which was limited to cropping, stitching multiple fields (64), rotating, and uniform contrast adjustments. Confocal micrographs are presented as single optical sections or maximum-intensity projections.

Uncolonized zebrafish bowel was measured using micrometer-calibrated gridded eyepieces. Mouse bowel colonization was measured with ImageJ. E18.5 bowel was considered hypoganglionic when gaps between myenteric ganglia became perceptibly larger and ganglia contained fewer neurons. For explant cultures, we measured the distance between gut edges and cell bodies of the most distant ENCDC in 8 sectors per explant. In $\mathrm{X}$-inactivation mosaic experiments, overall, $\mathrm{EGFP}^{+}$, and $\mathrm{EGFP}^{-}$migration were measured. Time-lapse images were acquired every 3 minutes on an AxioObserver.Z1 microscope (Zeiss) equipped with motorized stage, incubator, and $\mathrm{CO}_{2}$ controller. Cells were tracked with MTrackJ software (65). 
All experiments were performed in at least triplicate with separate embryos or separate pools of embryos in culture. For drug-treated pregnant mice, at least 3 litters were collected per treatment. Mean \pm SEM are plotted unless otherwise indicated. Analyses were performed by observers blind to genotype and treatment status.

Statistics. We used SigmaPlot 11 (Systat Software) or R (R Foundation) for analysis. For fetal bowel colonization, Student's $t$ test or 1-way ANOVA was used. For WT explants, repeated-measures ANOVA was used unless otherwise indicated. For mutant explants, 2-way ANOVA was used to test influence of genotype, treatment, and interactions. Paired $t$ tests or Wilcoxon signed-rank tests (when data were non-normally distributed) were used for mosaic analyses. Other parameters were compared with 1-way ANOVA, $t$ test, rank-sum test, or Kolmogorov-Smirnov test as indicated. For all analyses, $P<0.05$ was considered significant, and 2 -tailed tests were performed. $P$ values were adjusted for multiple comparisons by the Holm-Bonferroni procedure based on numbers of planned comparisons. When colonization was normalized to bowel length, absolute length of aganglionic (or abnormal) segments were also tested and always agreed with the normalized results.

Study approval. Animal experiments were approved by the Washington University Animal Studies Committee.

\section{Acknowledgments}

The authors thank Stephen Johnson and Matthew Goldsmith for invaluable assistance with zebrafish and the use of their facili- ties; the Chemical Genetics Screening Core (http://htc.wustl.edu) for the chemical library used in the screen; Beverly S. Mitchell for the gift of Hprtb-m3 mice; Michael Wegner and Michelle SouthardSmith for the gift of Sox $10^{\text {LacZ }}$ mice on the C3HeBFe/J background as well as helpful advice on genetic background; Sanjay Jain for the gift of $\operatorname{Ret}^{9}$ mice as well as helpful advice for selecting the appropriate Ret model; the Mouse Genetics Core (http://mgc.wustl.edu) for mutant mouse line maintenance; Craig Smith for the rabbit antiSoxE antibody; and Ming Fu, Hongtao Wang, Elizabeth WrightJin, Ellen Merrick Schill, Marina Avetisyan, and Shahriyar Majidi for help and support. Funding for this project was provided by the Children's Discovery Institute of Washington University and St. Louis Children's Hospital (grant nos. CH-II-1008-123 and CH-II2010-390), NIH grants RO1 DK087715 and RO1 DK057038, and a Burroughs Wellcome Fund Clinical Scientist Award in Translational Research (grant no. 1008525).

Received for publication March 8, 2013, and accepted in revised form August 12, 2013.

Address correspondence to: Robert O. Heuckeroth, The Children's Hospital of Philadelphia, Research Institute, 3615 Civic Center Blvd. Abramson Research Center - Suite 1116I, Philadelphia, Pennsylvania 19104-4318, USA. Phone: 314.286.2853; Fax: 314.286.2893; E-mail: HeuckerothR@email.chop.edu.
1. Heuckeroth RO. Hirschsprung disease. In: Faure C, Di Lorenzo C, Thapar N, eds. Pediatric Neurogastroenterology. New York, New York, USA: Humana Press; 2013:271-283.

2. Amiel J, et al. Hirschsprung disease, associated syndromes and genetics: a review. J Med Genet. 2008; 45(1):1-14.

3. Carrasquillo MM, McCallion AS, Puffenberger EG, Kashuk CS, Nouri N, Chakravarti A. Genomewide association study and mouse model identify interaction between RET and EDNRB pathways in Hirschsprung disease. Nat Genet. 2002;32(2):237-244.

4. Gabriel SB, et al. Segregation at three loci explains familial and population risk in Hirschsprung disease. Nat Genet. 2002;31(1):89-93.

5. Emison ES, et al. A common sex-dependent mutation in a RET enhancer underlies Hirschsprung disease risk. Nature. 2005;434(7035):857-863.

6. de Pontual L, et al. Epistatic interactions with a common hypomorphic RET allele in syndromic Hirschsprung disease. Hum Mutat. 2007;28(8):790-796.

7. Heanue TA, Pachnis V. Enteric nervous system development and Hirschsprung's disease: advances in genetic and stem cell studies. Nat Rev Neurosci. 2007;8(6):466-479.

8. Fu M, Tam PKH, Sham MH, Lui VCH. Embryonic development of the ganglion plexuses and the concentric layer structure of human gut: a topographical study. Anat Embryol. 2004;208(1):33-41.

9. Wallace AS, Burns AJ. Development of the enteric nervous system, smooth muscle and interstitial cells of Cajal in the human gastrointestinal tract. Cell Tissue Res. 2005;319(3):367-382.

10. Lake JI, Heuckeroth RO. Enteric nervous system development: migration, differentiation, and disease. Am J Physiol Gastrointest Liver Physiol. 2013; 305(1):G1-G24.

11. Vohra BPS, Fu M, Heuckeroth RO. Protein kinase $\mathrm{C} \zeta$ and glycogen synthase kinase- $3 \beta$ control neuronal polarity in developing rodent enteric neurons, whereas SMAD specific E3 ubiquitin protein ligase 1 promotes neurite growth but does not influence polarity. J Neurosci. 2007;27(35):9458-9468.

12. Fu M, et al. Vitamin A facilitates enteric nervous system precursor migration by reducing Pten accumulation. Development. 2010;137(4):631-640.

13. Stewart AL, Young HM, Popoff M, Anderson RB. Effects of pharmacological inhibition of small GTPases on axon extension and migration of enteric neural crest-derived cells. Dev Biol. 2007; 307(1):92-104.

14. Zhang Y, Kim T-H, Niswander L. Phactr4 regulates directional migration of enteric neural crest through PP1, integrin signaling, and cofilin activity. Genes Dev. 2012;26(1):69-81.

15. Anderson RB, Turner KN, Nikonenko AG, Hemperly J, Schachner M, Young HM. The cell adhesion molecule $\mathrm{L} 1$ is required for chain migration of neural crest cells in the developing mouse gut. Gastroenterology. 2006;130(4):1221-1232.

16. Breau MA, Dahmani A, Broders-Bondon F, Thiery J-P, Dufour S. $\beta 1$ integrins are required for the invasion of the caecum and proximal hindgut by enteric neural crest cells. Development. 2009;136(16):2791-2801.

17. Broders-Bondon F, Paul-Gilloteaux P, Carlier C, Radice GL, Dufour S. N-cadherin and $\beta 1$-integrins cooperate during the development of the enteric nervous system. Dev Biol. 2012;364(2):178-191.

18. Chong CR, Chen X, Shi L, Liu JO, Sullivan DJ. A clinical drug library screen identifies astemizole as an antimalarial agent. Nat Chem Biol. 2006;2(8):415-416.

19. Kuhlman J, Eisen JS. Genetic screen for mutations affecting development and function of the enteric nervous system. Dev Dyn. 2007;236(1):118-127.

20. Genentech Inc. CellCept. Genentech Web site. http://www.gene.com/download/pdf/cellcept prescribing.pdf. Accessed August 28, 2013.

21. Reagan-Shaw S, Nihal M, Ahmad N. Dose translation from animal to human studies revisited. FASEB J. 2008;22(3):659-661.

22. Sato Y, Heuckeroth RO. Retinoic acid regulates murine enteric nervous system precursor proliferation, enhances neuronal precursor differentiation, and reduces neurite growth in vitro. Dev Biol. 2008; 320(1):185-198.

23. Jonsson CA, Svensson L, Carlsten H. Beneficial effect of the inosine monophosphate dehydrogenase inhibitor mycophenolate mofetil on survival and severity of glomerulonephritis in systemic lupus erythematosus (SLE)-prone MRLlpr/lpr mice. Clin Exp Immunol. 1999;116(3):534-541.

24. Corpening JC, Deal KK, Cantrell VA, Skelton SB, Buehler DP, Southard-Smith EM. Isolation and live imaging of enteric progenitors based on Sox10-Histone2BVenus transgene expression. Genesis. 2011; 49(7):599-618.

25. Wallace AS, Schmidt C, Schachner M, Wegner M, Anderson RB. L1cam acts as a modifier gene during enteric nervous system development. Neurobiol Dis. 2010;40(3):622-633.

26. Uesaka T, Nagashimada M, Yonemura S, Enomoto H. Diminished Ret expression compromises neuronal survival in the colon and causes intestinal aganglionosis in mice. J Clin Invest. 2008; 118(5):1890-1898.

27. Simpson MJ, Zhang DC, Mariani M, Landman KA, Newgreen DF. Cell proliferation drives neural crest cell invasion of the intestine. Dev Biol. 2007; 302(2):553-568.

28. Hooper M, Hardy K, Handyside A, Hunter S, Monk M. HPRT-deficient (Lesch-Nyhan) mouse embryos derived from germline colonization by cultured cells. Nature. 1987;326(6110):292-295.

29. Allison AC, Eugui EM. Mycophenolate mofetil and its mechanisms of action. Immunopharmacology. 2000;47(2-3):85-118.

30. Hadjantonakis AK, Cox LL, Tam PP, Nagy A. An $\mathrm{X}$-linked GFP transgene reveals unexpected paternal $\mathrm{X}$-chromosome activity in trophoblastic giant cells of the mouse placenta. Genesis. 2001;29(3):133-140.

31. Anderka MT, Lin AE, Abuelo DN, Mitchell AA, Rasmussen SA. Reviewing the evidence for mycophenolate mofetil as a new teratogen: Case report and review of the literature. Am J Med Genet. 2009; 149A(6):1241-1248.

32. Lin AE, Singh KE, Strauss A, Nguyen S, Rawson $\mathrm{K}$, Kimonis VE. An additional patient with mycophenolate mofetil embryopathy: cardiac and facial analyses. Am J Med Genet A. 2011;155A(4):748-756.

33. Britsch S, et al. The transcription factor Sox 10 is a key regulator of peripheral glial development. Genes Dev. 2001;15(1):66-78. 
34. Paratore C, Eichenberger C, Suter U, Sommer L. Sox 10 haploinsufficiency affects maintenance of progenitor cells in a mouse model of Hirschsprung disease. Hum Mol Genet. 2002;11(24):3075-3085.

35. Young HM, et al. Dynamics of neural crest-derived cell migration in the embryonic mouse gut. Dev Biol. 2004;270(2):455-473.

36. Natarajan D, Marcos-Gutierrez C, Pachnis V, de Graaff E. Requirement of signalling by receptor tyrosine kinase RET for the directed migration of enteric nervous system progenitor cells during mammalian embryogenesis. Development. 2002; 129(22):5151-5160.

37. Gianino S, Grider JR, Cresswell J, Enomoto H, Heuckeroth RO. GDNF availability determines enteric neuron number by controlling precursor proliferation. Development. 2003;130(10):2187-2198.

38. Jain S, et al. Mice expressing a dominant-negative Ret mutation phenocopy human Hirschsprung disease and delineate a direct role of Ret in spermatogenesis. Development. 2004;131(21):5503-5513.

39. Kapur RP, Yost C, Palmiter RD. A transgenic model for studying development of the enteric nervous system in normal and aganglionic mice. Development. 1992;116(1):167.

40. Druckenbrod NR, Epstein ML. Age-dependent changes in the gut environment restrict the invasion of the hindgut by enteric neural progenitors. Development. 2009;136(18):3195-3203.

41. Hotta R, Anderson RB, Kobayashi K, Newgreen DF, Young HM. Effects of tissue age, presence of neurones and endothelin- 3 on the ability of enteric neurone precursors to colonize recipient gut: implications for cell-based therapies. Neurogastroenterol Motil. 2010;22(3):331-e86.

42. Curry CJ, et al. Smith-Lemli-Opitz syndrome-type II: multiple congenital anomalies with male pseudohermaphroditism and frequent early lethality. Am J Med Genet. 1987;26(1):45-57.

43. Barlow AJ, Dixon J, Dixon MJ, Trainor PA. Balancing neural crest cell intrinsic processes with those of the microenvironment in Tcof1 haploinsufficient mice enables complete enteric nervous system formation. Hum Mol Genet. 2012;21(8):1782-1793.
44. Long H, Cameron S, Yu L, Rao Y. De novo GMP synthesis is required for axon guidance in Drosophila. Genetics. 2006;172(3):1633-1642.

45. Schmidt F, Eckardt K, Shakibaei M, Glander P, Stahlmann R. Effects of mycophenolic acid alone and in combination with its metabolite mycophenolic acid glucuronide on rat embryos in vitro. Arch Toxicol. 2013;87(2):361-370.

46. Mondin M, Moreau V, Genot E, Combe C, Ripoche $\mathrm{J}$, Dubus I. Alterations in cytoskeletal protein expression by mycophenolic acid in human mesangial cells requires Rac inactivation. Biochem Pharmacol. 2007;73(9):1491-1498.

47. Krötz F, et al. Mycophenolate acid inhibits endothelial $\mathrm{NAD}(\mathrm{P}) \mathrm{H}$ oxidase activity and superoxide formation by a Rac1-dependent mechanism. Hypertension. 2007;49(1):201-208.

48. Kapur RP, Livingston R, Doggett B, Sweetser DA, Siebert JR, Palmiter RD. Abnormal microenvironmental signals underlie intestinal aganglionosis in dominant megacolon mutant mice. Dev Biol. 1996; 174(2):360-369.

49. Kapur RP, Sweetser DA, Doggett B, Siebert JR, Palmiter RD. Intercellular signals downstream of endothelin receptor-B mediate colonization of the large intestine by enteric neuroblasts. Development. 1995;121(11):3787-3795.

50. Carmona-Fontaine $\mathrm{C}$, et al. Complement fragment $\mathrm{C} 3$ a controls mutual cell attraction during collective cell migration. Developmental Cell. 2011; 21(6):1026-1037.

51. Carmona-Fontaine $\mathrm{C}$, et al. Contact inhibition of locomotion in vivo controls neural crest directional migration. Nature. 2008;456(7224):957-961.

52. Wang X, Chan AKK, Sham M-H, Burns AJ, Chan WY. Analysis of the sacral neural crest cell contribution to the hindgut enteric nervous system in the mouse embryo. Gastroenterology. 2011;141(3):992-1002.e6.

53. Anderson RB. Matrix metalloproteinase- 2 is involved in the migration and network formation of enteric neural crest-derived cells. Int J Dev Biol. 2010;54(1):63-69.

54. Murphey RD, Zon LI. Small molecule screening in the zebrafish. Methods. 2006;39(3):255-261.
55. Danielian PS, Muccino D, Rowitch DH, Michael SK, McMahon AP. Modification of gene activity in mouse embryos in utero by a tamoxifeninducible form of Cre recombinase. Curr Biol. 1998; 8(24):1323-1326.

56. Srinivas $S$, et al. Cre reporter strains produced by targeted insertion of EYFP and ECFP into the ROSA26 locus. BMC Dev Biol. 2001;1:4.

57. Enomoto H, Crawford PA, Gorodinsky A, Heuckeroth RO, Johnson EM Jr, Milbrandt J. RET signaling is essential for migration, axonal growth and axon guidance of developing sympathetic neurons. Development. 2001;128(20):3963-3974.

58. Stratman JL, Barnes WM, Simon TC. Universal PCR genotyping assay that achieves single copy sensitivity with any primer pair. Transgenic Res. 2003; 12(4):521-522.

59. Creedon DJ, et al. Neurturin shares receptors and signal transduction pathways with glial cell linederived neurotrophic factor in sympathetic neurons. Proc Natl Acad Sci USA. 1997;94(13):7018-7023.

60. Vohra BP, Planer W, Armon J, Fu M, Jain S, Heuckeroth RO. Reduced endothelin converting enzyme-1 and endothelin-3 mRNA in the developing bowel of male mice may increase expressivity and penetrance of Hirschsprung disease-like distal intestinal aganglionosis. Dev Dyn. 2007;236(1):106-117.

61. Wang H, Zhang Y, Heuckeroth RO. PAI-1 deficiency reduces liver fibrosis after bile duct ligation in mice through activation of tPA. FEBS Lett. 2007; 581(16):3098-3104.

62. Ramakers C, Ruijter JM, Deprez RHL, Moorman AF. Assumption-free analysis of quantitative realtime polymerase chain reaction (PCR) data. Neurosci Lett. 2003;339(1):62-66.

63. Pfaffl MW. A new mathematical model for relative quantification in real-time RT-PCR. Nucleic Acids Res. 2001;29(9):e45.

64. Preibisch S, Saalfeld S, Tomancak P. Globally optimal stitching of tiled 3D microscopic image acquisitions. Bioinformatics. 2009;25(11):1463-1465.

65. Meijering E, Dzyubachyk O, Smal I. Methods for cell and particle tracking. Methods Enzymol. 2012; 504:183-200. 